\title{
MEDICAL EQUIPMENTS AND ITS USES IN VARIOUS HOSPITALS AND CLINICS IN DHAKA BANGLADESH
}

\author{
Munima Haque ${ }^{1}$ \\ ${ }^{1}$ Department of EEE, Southeast University, Tejgaon Industrial Area, Tejgaon, Dhaka-1213, Bangladesh
}

\begin{abstract}
Biomedical imaging equipments like X-ray, CT (Computed Tomography), MRI (Magnetic Resonance imaging), Endoscopy, ECG (Electrocardiogram), EEG (Electroencephalography) and many other imaging instruments are being used in our country every day for medical diagnosis purposes. In this research various public and private hospitals/clinics in Dhaka city were investigated and it was found that every hospital has X-ray and ultrasound machine and some hospital do not have CT or MRI machine. So the percentage of X-ray and Ultrasound machine is $100 \%$ and another CT or MRI machine is $90 \%$ available on our researched hospital in Dhaka city. The patients in Dhaka city and also those who comes for treatment from outside Dhaka have accessibility to the medical equipments in Dhaka. But in private hospitals/clinics the medical equipment services are expensive. The public hospitals provide cheaper service and so more cost effective medical services needs to be implemented for patients.
\end{abstract}

Keywords: Medical Imaging, Instruments, Hospitals, Clinics, Applications, Cost, Dhaka

\section{INTRODUCTION}

Biomedical imaging is one of the most important subjects in all over the world. Its demand is increasing day by day not only to the world, but also in Bangladesh. Medical imaging was primarily seen as the use of non-invasive techniques to create internal images of the human body for clinical or medical purposes. There is a continuous drive not only to improve the diagnostic yield of medical imaging techniques for clinical use, but also the management of the huge amount of digital information available to medical imaging departments. Today there is an increasing interest to harness medical imaging processes to improve research in the biophysical domain. The rapid changes in digital technology have also created opportunities for the development of hightech equipment employed in medical imaging practice. Many different techniques have been developed to get a look inside the patient. They are all based on a signal travelling right through a patient: different kinds of electromagnetic radiation (such as X-rays or gamma-rays) or sound waves. These signals interact with the tissues of the patient. By detecting the signal coming out of the body an image of the inside of the patient can be made. In this way the patient seems to become transparent.

In last few decades, the rapid progress in Medical imaging techniques has completely revolutionized medical diagnostic system. The modern imaging system is not only limited to image production, but also focused on image processing, image transmission, image recording and automated diagnosis. In order to give best treatment doctor needs to examine patient's body from inside. Medical imaging system reveals internal organs hidden by skin and creates visual representation of internal body for diagnosis purpose.
Earlier the interpretation of medical images was done by physicians manually i.e. doctor had only 5 senses to examine problem but nowadays computer aided diagnosis system acts as an intermediate between doctor and patient, the machine examine patients body and doctor examines the report generated by machine which gives fast, accurate , reliable and consistent results.

Medical imaging has advanced in remarkable ways since the discovery of x-rays. Using X-ray, one can image the human body in intricate detail using computed tomography, magnetic resonance imaging, positron emission tomography, ultrasound, and various other instruments. Such technology allows for improved screening, diagnosis, and monitoring of disease. (Scatliff, 2014) There are various medical imaging techniques and applications (Hodder, 2012) Kunio in a review article has discussed about Diagnostic imaging over the last 50 years: research and development in medical imaging science and technology. (Kunio, 2006). The use of imaging has skyrocketed in the past decade, but no one patient population or medical condition is responsible. (Smith-Bindman, 2008)

Today, medical images are obtained by different acquisition modalities, including X-ray Computed Tomography (CT), Magnetic Resonance Imaging (MRI), single photon emission tomography (SPECT), positron emission tomography (PET), ultrasounds etc. Forming images of the human body using ionizing radiation has been a part of the diagnostic process in medicine for many years. More recently, other probing radiations, notably ultrasound, have provided successful diagnostic images. Tomography is now in widespread use in the clinic with ionizing radiations (Xray, isotopes) and with nuclear magnetic resonance imaging. 
In the past few years, other probing radiations have been considered for tomography, including ultrasound (Mueller, 1979) (Duchene, 1985), very low-frequency electromagnetic fields in electrical impedance tomography (Barber, 1983), and microwaves (Bolomey, 1982), (Ermert, 1986).

Nowadays biomedical imaging is one of the most developing research sectors all over the world. Famous researchers in many countries work in this field. Germany, USA, Canada, Sweden and many other countries work on medical imaging. In Bangladesh, recently some research and education processing of biomedical engineering has been started. Some university has opened undergraduate program on biomedical engineering. Recently some professors of BUET and DMC discovered Elastrography which has been accepted by world's scientists. Elastrography helps to detect breast cancer. (Nakata, 2014)

The current population of Bangladesh is 162,956,972 as of Monday, July 11, 2016, based on the latest United Nations estimates. Bangladesh population is equivalent to about $2.19 \%$ of the total world population. In terms of population, Bangladesh ranks number 8 in the list (BD, 2016). Dhaka, the capital of Bangladesh has a population of 1, 25, 17, 361 people having 68, 13, 463 males and 57, 03, 908 females. $70.50 \%$ of Dhaka city population are educated. Per capita income is 550 USD. (Dhaka, 2016)

\section{METHODS}

Questionnaire forms of hospital/clinic features and medical equipment usage info were prepared and used for investigation. Permission was taken for access to the hospital/clinics imaging divisions. Questions were asked and by observation of the instruments, the formed were completed.

\section{RESULTS}

Findings at different EMF sources:Public hospitals features. Table 1 shows the public hospitals in Dhaka and its features and divisions. Shaheed Suhrawardy Medical College \& Hospital is a government hospital where patient can undergo treatment with very low cost. Dhaka Medical College \& Hospital is one of the busiest hospitals in Dhaka city. Some Patient uses floor for getting treatment, critical patient is also treated here. Bangabandhu Sheikh Mujib Medical University is the first medical university in Bangladesh where doctor takes their master's degree from here and it has four blocks (A, B, C, D) building with a large area. Two new buildings are also established for outgoing patient treatment. BIRDEM Cardiac Hospital is mainly diabetes basis hospital but all types of patient are treated here. The patient pressure is so much in this hospital.

Private hospitals features
Table 2A shows the private hospitals/clinics in Dhaka and its features and divisions. IBN-SINA Medical College \& Hospital Ibn Sina is the mother of treatment. Basically this hospital name has come from this and it has more branches in Dhaka city, main branch is situated in Dhanmondi, Dhaka. Square Hospital Limited is the number one private hospital in Bangladesh and its service is really much better than another hospital. The internal environment is very neat and clean of this hospital and the staff behave is really good. Gastro Liver Hospital \& Research Center is a well-designed modern hospital which was established recently. In Labaid Hospital \& Diagnostic Center the environment is nice and the staffs are well dressed. On the roof of the hospital building have a nice garden and also have a canteen. At the Popular Medical college Hospital \& Consultation Centre the hospital building is nice looking and also have the patient pressure as it is main branch. At Ad-din Womens Medical \& Hospital the environment of the hospital is very neat and clean. The cost is not so high, the hospital have a foundation named Ad-din foundation. This is basically pregnant women and child care basis hospital. Dr. Sirazul Islam Medical College \& Hospital is a new medical college and hospital so patient pressure is not so much but imaging equipments are new.

Table 2B shows the private hospitals/clinics in Dhaka and its features and divisions. Administration of this hospital is very good. Day by day this hospital is improving their infrastructure. They are giving their best service. DigiLab is the only hospital where separate treatment units are for female and male patients. NARGIS Clinic seems to be very good. There are various kinds of equipments and the housing location is very nice. The maintenance of Popular Hospital Sector-10, Dhaka is very good. The hospital authority takes responsibility with the cleanliness. The administration of Khidmah Hospital is very good. It is improving day by day. Also, it gives discounts to the poor patients. Apollo Hospital is the only 'JCI Accredited' multidisciplinary super specially tertiary care hospital in Bangladesh which its operation on 2005 with a mission to provide high quality standard health care with latest medical, surgical and diagnostic facilities that meets the needs and exceeds the expectations of peoples of Bangladesh. Apollo Hospital is now financially robust with a healthy balance. The cash flow of the hospital is also improving. They invest in equipment and for recruiting and retaining skilled health care workers to give the proper treatment. Labaid Hospital Gulshan2 its hospitality system is very good. They have many kinds of equipments and their housing location is very good. 
Table 1: Hospital/clinic features, units/divisions and diagnostic Imaging Equipments available in various public Hospital \& clinics at Dhaka city in Bangladesh

\begin{tabular}{|c|c|c|c|c|}
\hline No. & $\begin{array}{l}\text { Hospital/Clinic } \\
\text { name }\end{array}$ & $\begin{array}{l}\text { Features (No. } \\
\text { of beds, No. of } \\
\text { patients } \\
\text { treated, No. of } \\
\text { doctors) }\end{array}$ & Units/division & Imaging equipments \\
\hline 1 & $\begin{array}{l}\text { Shaheed } \\
\text { Suhrawardy } \\
\text { Medical College \& } \\
\text { Hospital SSMCH) } \\
\text { (College Gate, } \\
\text { Sher-e-Bangla } \\
\text { Nagar, Dhaka) }\end{array}$ & $\begin{array}{l}\text { Beds : } 850 \\
\text { Patient treated } \\
\text { per day : } 1500- \\
2000 \quad \text { (in } \\
\text { summer } \\
\text { season), } 1200- \\
1600 \text { (in winter } \\
\text { season) } \\
\text { Doctors : } 167\end{array}$ & $\begin{array}{l}\text { Units/Division : } 22 \\
\text { These are - Medicine, Orthopedic, Oncology, } \\
\text { Breast feeding, Dermatology, Emergency, } \\
\text { Child division, Surgery, Physical medicine, } \\
\text { Gynecology, Hematology, Neurology, } \\
\text { Cardiology, Dental, Urology, Mental Health } \\
\text { division, Biomedical \& Biotechnology division, } \\
\text { ENT division, Eye division, Respiratory } \\
\text { medicine division,, Burn Plastic\& } \\
\text { Reconstructive surgery division. }\end{array}$ & $\begin{array}{l}\text { X-Ray, } \\
\text { Ultrasonography, } \\
\text { Endoscopy, } \\
\text { CT(Computed } \\
\text { Tomography) Scan \& } \\
\text { MRI(Magnetic } \\
\text { Resonance Imaging) }\end{array}$ \\
\hline 2 & $\begin{array}{l}\text { Dhaka Medical } \\
\text { College \& Hospital } \\
\text { DMCH) (Dhaka } \\
\text { University Area) }\end{array}$ & $\begin{array}{l}\text { Beds : } 1200 \\
\text { Patient treated } \\
\text { per day : 4000- } \\
5000(\text { In } \\
\text { summer), } \\
3500-4000(\text { In } \\
\text { winter) } \\
\text { Doctors : } \\
\text { Above } 600\end{array}$ & $\begin{array}{l}\text { Units/Division : } 27 \\
\text { Internal Medicine, Cardiology, Nephrology, } \\
\text { Gastroenterology, Endocrinology, Hematology, } \\
\text { Neurology, Dermatology, Mental, Physical } \\
\text { Medicine, Child Division, General surgery, } \\
\text { Urology, Oncology, Dental, Pedicardiac } \\
\text { surgery, Orthopedic, ENT, Head \& Neck, Eye } \\
\text { Division, Cancer \& Radiotherapy, Burn \& } \\
\text { Plastic Surgery, Gynee \& Obs, Anesthesiology, } \\
\text { Emergency, Blood Transfusion, Clinical } \\
\text { Pathology \& Radiology Imaging. }\end{array}$ & $\begin{array}{l}\text { X-Ray, } \\
\text { Ultrasonography, } \\
\text { Endoscopy, CT, MRI, } \\
\text { Mammography, } \\
\text { ECG(Electrocardiogram) }\end{array}$ \\
\hline 3 & $\begin{array}{l}\text { Bangabandhu } \\
\text { Sheikh Mujib } \\
\text { Medical University } \\
\text { BSMMU) } \\
\text { (Shahbag, Dhaka) }\end{array}$ & $\begin{array}{l}\text { Beds : } 1600 \\
\text { Patient treated } \\
\text { per day : 4000- } \\
5000 \text { (in } \\
\text { summer } \\
\text { season), } \\
4000-4600 \text { (in } \\
\text { winter season) } \\
\text { Doctors : } \\
\text { Above } 700\end{array}$ & $\begin{array}{l}\text { Units/Division : } 27 \\
\text { Medicine,Gastro Aentralogy, Androcrinology, } \\
\text { Neuro Medicine, Child neurology, Oncology, } \\
\text { Cardiac surgery \& Mental division, Physical } \\
\text { Medicine, Clinical pathology, Orthopaedix, } \\
\text { Breast feeding, Dermatology, Emergency, } \\
\text { Child division, Anesthesia, Surgery, } \\
\text { Gynecology, Hematology, Neurology, } \\
\text { Nephrology, Cardiology, Dental, Urology, } \\
\text { Mental Health division, Biomedical \& } \\
\text { Biotechnology division, ENT division, Eye } \\
\text { division, Respiratory medicine division, Burn } \\
\text { Plastic\& Reconstructive surgery division. }\end{array}$ & $\begin{array}{l}\text { X-Ray, } \\
\text { Ultrasonography, } \\
\text { Endoscopy, CT, } \\
\text { Mammography }\end{array}$ \\
\hline 4 & $\begin{array}{l}\text { Birdem Cardiac } \\
\text { Hospital (Shahbag, } \\
\text { Dhaka) }\end{array}$ & $\begin{array}{l}\text { Beds : } 992 \\
\text { Patient treated } \\
\text { per day: } 3000- \\
3500 \\
\text { Doctors : } 500\end{array}$ & $\begin{array}{l}\text { Units/Division : } 27 \\
\text { Medicine, Gastro Aentralogy, Androcrinology, } \\
\text { Neuro Medicine, Child neurology, oncology, } \\
\text { Cardiac surgery \& Mental division, Physical } \\
\text { Medicine, Clinical pathology, Orthopedic, } \\
\text { Breast feeding, Dermatology, Imargency, Child } \\
\text { division, Anesthesia, Surgery, Gynecology, } \\
\text { Hepatology, Neurology, Nephrology, } \\
\text { Cardiology, Dental, Urology, Mental Health } \\
\text { division, Biomedical \& Biotechnology division, } \\
\text { ENT division, Eye division, Respiratory } \\
\text { medicine division, Burn Plastic\& } \\
\text { Reconstructive surgery division. }\end{array}$ & $\begin{array}{l}\text { X-Ray, } \\
\text { Ultrasonography, } \\
\text { Endoscopy, CT, MRI, } \\
\text { Mammography }\end{array}$ \\
\hline
\end{tabular}


Table 2A: Hospital/clinic features, units/divisions and diagnostic Imaging Equipments available in various private Hospital \& clinics at Dhaka city in Bangladesh

\begin{tabular}{|c|c|c|c|c|}
\hline No. & Hospital/Clinic name & $\begin{array}{l}\text { Features (No. } \\
\text { of beds, No. } \\
\text { of patients } \\
\text { treated, No. of } \\
\text { doctors) }\end{array}$ & Units/division & $\begin{array}{l}\text { Imaging } \\
\text { equipments }\end{array}$ \\
\hline 5 & $\begin{array}{l}\text { IBN-SINA } \\
\text { MedicalCollege\&Hospital } \\
\text { (Kallyanpur, Mirpur, } \\
\text { Dhaka) }\end{array}$ & $\begin{array}{l}\text { Beds : } 250 \\
\text { Patient treated } \\
\text { per day : } 600- \\
700 \text { Doctors : } \\
45\end{array}$ & $\begin{array}{l}\text { Units/Division : 13These are- Medicine, } \\
\text { Gastroenterology, Surgery division, Gyne \& obs, } \\
\text { ENT division, Orthopedic, Eye division, Child \& } \\
\text { child neurology division, Dermatology, Monorog, } \\
\text { Cardiology, Urology }\end{array}$ & $\begin{array}{l}\text { X-Ray, } \\
\text { Ultrasonography, } \\
\text { Endoscopy }\end{array}$ \\
\hline 6 & $\begin{array}{l}\text { Square Hospital Limited } \\
\text { (Green road, Dhanmondi, } \\
\text { Dhaka) }\end{array}$ & $\begin{array}{l}\text { Beds : } 450 \\
\text { Patient treated } \\
\text { per day : } 800- \\
1000 \\
\text { Doctors : } 86\end{array}$ & 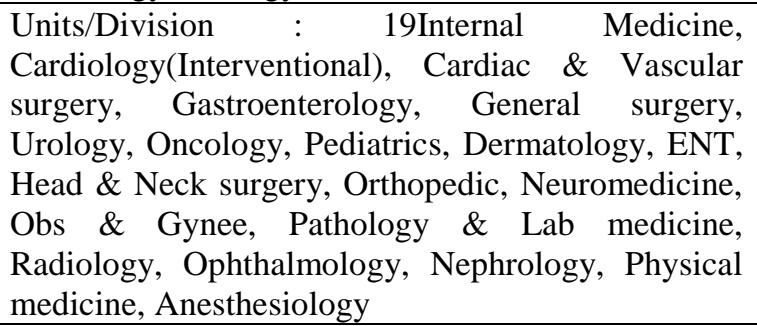 & $\begin{array}{l}\text { X-Ray, } \\
\text { Ultrasonography, } \\
\text { Endoscopy, CT, } \\
\text { MRI }\end{array}$ \\
\hline 7 & $\begin{array}{l}\text { Gastro Liver Hospital \& } \\
\text { Research center (Green } \\
\text { road, Dhanmondi, Dhaka) }\end{array}$ & $\begin{array}{l}\text { Beds : } \\
500 \text { (Five } \\
\text { hundred) } \\
\text { Patient treated } \\
\text { per day : 500- } \\
600 \text { Doctors : } \\
95\end{array}$ & 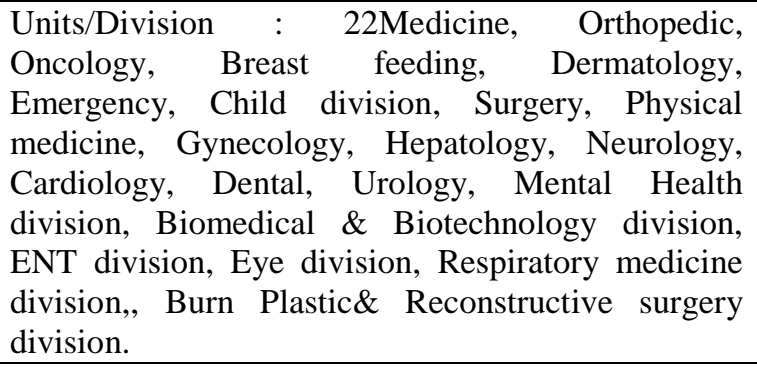 & $\begin{array}{l}\text { X-Ray, } \\
\text { Ultrasonography, } \\
\text { Endoscopy, CT, } \\
\text { MRI }\end{array}$ \\
\hline 8 & $\begin{array}{l}\text { Labaid Hospital \& } \\
\text { Diagnostic Center } \\
\text { (Dhanmondi, Dhaka) }\end{array}$ & $\begin{array}{l}\text { Beds : } 400 \\
\text { Patient treated } \\
\text { per day : } 800- \\
\text { 1000Doctors : } \\
\text { Full time- } 48, \\
\text { Part time-103, } \\
\text { Friday-10 }\end{array}$ & $\begin{array}{l}\text { Units/Division : 20Interventional Cardiology, } \\
\text { General \& Laproscopic, Nephrology, Neurology, } \\
\text { Urology \& Andrology, Gynecology, Oncology, } \\
\text { Internal Medicine, Trauma \&Orthopedic, ENT } \\
\text { Division, Dermatology, Dental Division, } \\
\text { Gastroenterology, Pediatric \& Neonatology, } \\
\text { Physical Medicine, Burn \& Plastic, Endrocrinology, } \\
\text { Child division, Urology, Hematology Division. }\end{array}$ & $\begin{array}{l}\text { Imaging } \\
\text { Equipments: X- } \\
\text { Ray, } \\
\text { Ultrasonography, } \\
\text { Endoscopy, CT, } \\
\text { MRI }\end{array}$ \\
\hline 9 & $\begin{array}{l}\text { Popular Medical college } \\
\text { Hospital \& Consultation } \\
\text { Centre } \\
\text { Dhaka) }\end{array}$ & $\begin{array}{l}\text { Beds : } 350 \\
\text { Patient treated } \\
\text { per day : } 700- \\
800 \\
\text { Doctors : } 114\end{array}$ & 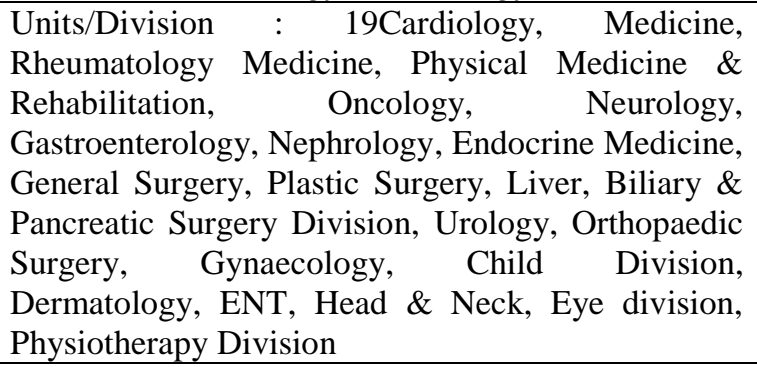 & $\begin{array}{l}\text { X-Ray, } \\
\text { Ultrasonography, } \\
\text { Endoscopy, CT }\end{array}$ \\
\hline 10 & $\begin{array}{l}\text { Ad-din Womens Medical } \\
\& \quad \text { Hospital (Boro } \\
\text { Moghbazar, Dhaka) }\end{array}$ & $\begin{array}{l}\text { Beds : } 200 \\
\text { Patient treated } \\
\text { per day : } 500- \\
600 \text { Doctors : } \\
62\end{array}$ & $\begin{array}{l}\text { Units/Division : } 15 \text { Medicine, Surgery, Gynee \& } \\
\text { obs, Child division, Orthopedic \& Trauma, Urology, } \\
\text { Child Surgery, ENT Division, Eye Division, Dental, } \\
\text { Dermatology, Anesthesiology, Radiology, } \\
\text { Pathology \& Microbiology Division. }\end{array}$ & $\begin{array}{l}\text { X-Ray, } \\
\text { Ultrasonography, } \\
\text { ECG, EEG, CT }\end{array}$ \\
\hline 11 & $\begin{array}{l}\text { Dr. Sirazul Islam Medical } \\
\text { College \& } \\
\text { Hospital(Malibagh, } \\
\text { Dhaka) }\end{array}$ & $\begin{array}{l}\text { Beds : } 250 \\
\text { Patient treated } \\
\text { per day : } 100- \\
150 \text { Doctors : } \\
65\end{array}$ & $\begin{array}{l}\text { Units/Division : 18Medicine, Cardiology, } \\
\text { Gynecology, Nephrology, Neuromedicine, Physical } \\
\text { medicine, Eye division, Child division, Dental, } \\
\text { General Surgery, Urology, Cancer, Orthopaedic, } \\
\text { Radiology \& Imaging, Dermatology, Emergency, } \\
\text { Neurology, ENT division. }\end{array}$ & $\begin{array}{l}\text { X-Ray, } \\
\text { Ultrasonography, } \\
\text { Endoscopy, CT, } \\
\text { MRI }\end{array}$ \\
\hline
\end{tabular}


Table 2B. Hospital/clinic features, units/divisions and diagnostic Imaging Equipments available in various private Hospital \& clinics at Dhaka city in Bangladesh (contd. from Table 2A)

\begin{tabular}{|c|c|c|c|c|}
\hline No. & $\begin{array}{l}\text { Hospital/Clinic } \\
\text { name }\end{array}$ & $\begin{array}{l}\text { Features (No. of beds, No. } \\
\text { of patients treated, No. of } \\
\text { doctors) }\end{array}$ & Units/division & Imaging equipments \\
\hline 12 & $\begin{array}{l}\text { Islami Bank } \\
\text { Central } \\
\text { Hospital(Kakrail, } \\
\text { Dhaka) }\end{array}$ & $\begin{array}{l}\text { Beds : } 160 \text { (total)ICU beds: } \\
\text { 26Doctors : } 50 \text { up }\end{array}$ & $\begin{array}{l}\text { Units/Division: Two buildings } \\
\text { having } 19 \text { floors. }\end{array}$ & $\begin{array}{l}\text { X-Ray, CT, } r \text { ICU } \\
\text { ventilator,Ultrasonography, } \\
\text { Haemodialysis, Syringe } \\
\text { pump }\end{array}$ \\
\hline 13 & $\begin{array}{l}\text { National Heart } \\
\text { Foundation } \\
\text { Hospital, Mirpur, } \\
\text { Dhaka }\end{array}$ & $\begin{array}{lcr}\text { Beds : } & \text { 300ICU } & \text { beds: } \\
\text { 16Doctors } & : & 75- \\
\text { 80Consultant doctors: } & \text { 30- } \\
\text { 35Nurses: } 450 & & \end{array}$ & - & \begin{tabular}{lr} 
X-Ray, & \multicolumn{2}{c}{ Ultrasono, } \\
Endoscopy, CT, MRI, \\
ECG, echo
\end{tabular} \\
\hline 14 & $\begin{array}{l}\text { DigiLab Medical } \\
\text { Services Ltd., } \\
\text { Mirpur, Dhaka }\end{array}$ & $\begin{array}{l}\text { Beds : } 500 \text { roomsPatient } \\
\text { treated }>1 \text { lac Doctors : } 31\end{array}$ & $\begin{array}{l}\text { Units/Division: } 5 \text { units in } \\
\text { country. } \\
\text { Free: diabetic care center, } \\
\text { consultation services for the } \\
\text { poor, ambulance service for CT } \\
\text { patient, Special discount for } \\
\text { corporate patient, Female } \\
\text { separate unit for all tests }\end{array}$ & $\begin{array}{l}\text { X-Ray, Ultrasonography, } \\
\text { Endoscopy, CT, MRI }\end{array}$ \\
\hline 15 & $\begin{array}{l}\text { Islamia } \quad \text { General } \\
\text { Hospital, } \\
\text { Jatrabari, }\end{array}$ & $\begin{array}{l}\text { Beds : 30Patient treated per } \\
\text { day : 20Doctors : } 30\end{array}$ & - & - \\
\hline 16 & $\begin{array}{l}\text { Monowara General } \\
\text { Hospital, } \\
\text { Biswaroad }\end{array}$ & $\begin{array}{l}\text { Beds : 100Patient treated } \\
\text { per day }: \text { 80Doctors }: 50\end{array}$ & - & - \\
\hline 17 & $\begin{array}{lr}\text { Al-Karim } & \text { General } \\
\text { Hospital, } & \text { North } \\
\text { Jatrabari, Dhaka }\end{array}$ & $\begin{array}{l}\text { Beds : 100Patient treated } \\
\text { per day : } 120 \text { Doctors : 45- } \\
50\end{array}$ & - & - \\
\hline 18 & $\begin{array}{l}\text { Nargis } \quad \text { Clinic, } \\
\text { Sherpur }\end{array}$ & $\begin{array}{l}\text { Bed: } 100 \text {, No ICU bedNo. } \\
\text { of doctor: } 30 \text {, Outdoor bed: } \\
40\end{array}$ & - & $\begin{array}{l}\text { X-ray, MRI, Ultrasono, } \\
\text { endoscopy, ECG }\end{array}$ \\
\hline 19 & $\begin{array}{l}\text { Popular Hospital, } \\
\text { Sector-10,Dhaka }\end{array}$ & $\begin{array}{l}\text { Bed: } 135, \quad \text { ICU bed: } \\
\text { 20Outdoor beds: } 40 \text {, Docs: } \\
\text { 30+ }\end{array}$ & - & $\begin{array}{l}\text { X-ray, Ultrasonography, } \\
\text { endoscopy, ECG }\end{array}$ \\
\hline 20 & $\begin{array}{l}\text { Ayesha Memorial, } \\
\text { Mokakhali }\end{array}$ & $\begin{array}{l}\text { Beds: 220, ICU beds: } 15 \text {, } \\
\text { NICU: } 18, \quad \text { Normal: } \\
\text { 87Doctors: } 40\end{array}$ & 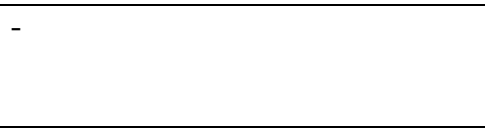 & $\begin{array}{ll}\text { X-ray, } & \text { MRI, } \\
\text { Ultrasonography, } \\
\text { endoscopy, ECG, CT }\end{array}$ \\
\hline 21 & $\begin{array}{l}\text { Khidmah Hospital } \\
\text { Pvt. Ltd. }\end{array}$ & $\begin{array}{l}\text { Beds: } 75 \text { (total)VIP bed: } 10 \text {, } \\
\text { Other bed: 65Doctor: } 40+\end{array}$ & $\begin{array}{l}\text { Internal medicine, respiratory, } \\
\text { day care, surgical unit, etc. }\end{array}$ & $\begin{array}{l}\text { X-ray, Syringe pump, } \\
\text { Ultrasonography, } \\
\text { endoscopy, CT etc. }\end{array}$ \\
\hline 22 & $\begin{array}{lr}\text { Badda } & \text { General } \\
\text { Hospital, } & \text { Uttar } \\
\text { Badda, } & \\
\text { Gulshan1,Dhaka } \\
\end{array}$ & $\begin{array}{l}\text { Beds: 50, Special bed: } \\
\text { 15Consultant } \\
\text { 28Specialist: } 20 \text { doctors: } \\
\text { duty: } 5\end{array}$ & - & 50 equipments \\
\hline 23 & $\begin{array}{l}\text { Pancare Hospital } \\
\text { Ltd. Dhanmondi } \\
\text { R/A, Dhaka }\end{array}$ & $\begin{array}{l}\text { Floor: 10NICU: 10, Beds: } \\
\text { 20NICU beds: } 10\end{array}$ & $\begin{array}{l}3 \text { doctors for each shift along } \\
\text { with NICU. Each shift consists } \\
\text { of } 8 \text { hours }\end{array}$ & ( \\
\hline 24 & $\begin{array}{lr}\text { Aryan } & \text { General } \\
\text { Hospital } & \& \\
\text { Diagnostic } & \text { center, } \\
\text { Khilkhet } & \\
\end{array}$ & $\begin{array}{l}\text { No beds } \\
\text { Doctors: } 35\end{array}$ & - & $\begin{array}{l}\text { X-ray, } \quad \text { Haemodialysis } \\
\text { Ultrasonography, etc. }\end{array}$ \\
\hline 25 & $\begin{array}{l}\text { Dogma Diagnostic } \\
\text { center, Uttar Badda }\end{array}$ & No bedsDoctors: $\sim 45$ & - & $\begin{array}{l}\text { X-ray, Haemodialysis } \\
\text { Ultrasonography, etc. }\end{array}$ \\
\hline 26 & $\begin{array}{l}\text { United Hospital, } \\
\text { Gulshan2, Dhaka }\end{array}$ & $\begin{array}{l}\text { Beds: } 400 \text { total } \\
\text { ICU beds: } 20 \text {, Other beds: } \\
380 \\
\text { Doctors: } 100-120 \text { (MBBS) }\end{array}$ & - & - \\
\hline
\end{tabular}




\begin{tabular}{|c|c|c|c|c|}
\hline & & Consultants: 60 & & \\
\hline 27 & $\begin{array}{l}\text { Apollo Hospital, } \\
\text { Bashundhara R/A, } \\
\text { Dhaka }\end{array}$ & $\begin{array}{l}\text { Beds: } 450 \\
\text { Doctors: } 54 \text { up } \\
\text { Patients treated/day: } 1200- \\
1500 \\
\text { ICU: (1) MICU: } 18 \text { beds (2) } \\
\text { SICU: } 9 \text { beds (3) HDU: } 12 \\
\text { beds (4) CTHDU: } 5 \text { beds } \\
\text { (5) KTU: } 2 \text { beds (6) NICU: } \\
12 \text { beds (7) CCU: } 11 \text { beds } \\
\text { (8) ICCU: } 8 \text { beds (9) } \\
\text { CTICU: } 7 \text { beds (10) NICU: } \\
16 \text { beds. }\end{array}$ & 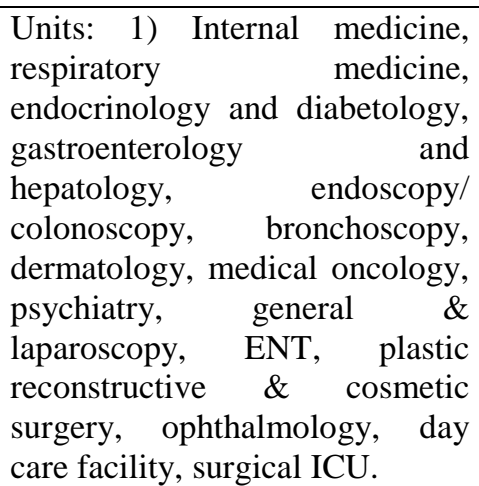 & - \\
\hline 28 & $\begin{array}{l}\text { Labaid Hospital, } \\
\text { Gulshan2, Dhaka }\end{array}$ & $\begin{array}{l}\text { Bed: } 65 \text { total. ICU bed: } 10 \text {, } \\
\text { Outdoor beds: } 15 \text { Doctor: } 35\end{array}$ & - & $\begin{array}{l}\text { MRI, ECG, } \\
\text { endoscopy, ultrasonogram }\end{array}$ \\
\hline 29 & $\begin{array}{lr}\text { IBN } & \text { SINA } \\
\text { Diagnostic } & \text { and } \\
\text { Consultation } & \\
\text { Center, } & \text { Uttar } \\
\text { Badda, Dhaka } & \end{array}$ & $\begin{array}{l}\text { No beds this center } \\
\text { Doctors: } 70\end{array}$ & No units & X-ray, CT scan, dental etc. \\
\hline
\end{tabular}

Public hospitals available equipments and uses

Table 3 shows the public hospitals in Dhaka and its medical equipments and applications. In Shaheed Suhrawardy Medical College \& Hospital, to collect this information we faced a beautiful experience about medical imaging instruments and the condition of the hospital in our Dhaka city. Dhaka Medical College and Hospital: Beside the all imaging equipment room have an alert notice about radiation problem and entry is restricted. In Bangabandhu Sheikh Mujib Medical University, all imaging equipment rooms have an alertment notice/info about radiation problem and entry is restricted for everyone. In Birdem Cardiac Hospital imaging equipments are new and the operators give us information intelligently.

Private hospitals/clinics available equipments and uses Table 4 show the private hospitals/clinics in Dhaka and its medical equipments and applications. In every diagnostic exam IBN-SINA Medical College \& Hospital provides $25 \%$ discount and the patient gets this opportunity from all branches. In Square Hospital Limited the treatment cost is so high. Mainly rich people take the treatment from this hospital. The entire machine are new and the operators behave is quite good and they operate machine nicely. Gastro Liver Hospital \& Research Center is a well-designed imaging room and the examinee patient wait outside of the imaging room because of radiation problem. In Labaid Hospital \& Diagnostic Center the staffs and operators are helpful and sometimes the doctors operate the machine like CT, MRI and Endoscopy. At the Popular Medical college Hospital \& Consultation Centre the Imaging equipments were looking new but diagnosis cost is not comfortable for all patient specially the poor patient. At the Ad-din Womens Medical \& Hospital, though this is women hospital so it became hard to collect the information but instrument is really good and the staff behavior is also good. Meanwhile in this hospital we see the different looks where the majority staffs are women. At Dr. Sirazul Islam Medical College \& Hospital, the imaging equipments are new but patient pressure is not so much because it is new hospital. The operator's behaviors are also remarkable. Badda General Hospital has more equipments but most of them are old. Apollo hospital has a wide range of equipment services but access wasnot available for information. Pancare Hospital Ltd. Is a small but efficient hospital and all equipments are in good condition.

In most cases, X-rays imaging instrument was used for detecting block of lungs, fracture of bones, fracture of hurt blockage. The Ultrasound machine was mainly used for pregnant women to show the condition unborn baby. Also used for kidney patient. CT scan was used for detecting the brain damage. The MRI was used for scanning the whole body to detect neurological and brain problem. Mammography instrument was used for detecting breast cancer and Endoscopy machine was used for finding ulcer and gastric problems. 
Table 3: Equipments available and its uses in various public hospitals in Dhaka

\begin{tabular}{|c|c|c|c|c|c|}
\hline No. & $\begin{array}{l}\text { Hospital/clinic } \\
\text { name }\end{array}$ & Equipment Name & $\begin{array}{lrr}\text { Equipment } & \text { info. (Machine, } \\
\text { Machine } & \text { model, } & \text { made } \\
\text { country, } & \text { Date } & \text { of } \\
\text { installation) } & & \end{array}$ & $\begin{array}{ll}\text { Application/Uses } & \text { for } \\
\text { diagnostic purposes } & \end{array}$ & Observations \\
\hline \multirow[t]{5}{*}{1} & \multirow[t]{5}{*}{$\begin{array}{l}\text { Shaheed } \\
\text { Suhrawardy } \\
\text { Medical College } \\
\text { \& Hospital }\end{array}$} & X-ray & $\begin{array}{l}\text { Machine: } \\
\text { ToshibaModel:DMT- } \\
\text { 80Made country: } \\
\text { KoreaInstall date:5-09-05 }\end{array}$ & $\begin{array}{l}\text { Uses for bio and non bio } \\
\text { medical diagnostic purpose }\end{array}$ & $\begin{array}{l}\text { It looks old and the } \\
\text { operators faces } \\
\text { some problem at } \\
\text { that time }\end{array}$ \\
\hline & & Ultrasonography & $\begin{array}{ll}\text { Machine: } & \\
\text { ToshibaModel:DC-70Made } \\
\text { country: } & \text { JapanInstall } \\
\text { date:2-09-12 } & \end{array}$ & $\begin{array}{l}\text { Mainly used for pregnant } \\
\text { women to know the baby's } \\
\text { position }\end{array}$ & $\begin{array}{l}\text { The operators did } \\
\text { not allow entry }\end{array}$ \\
\hline & & CT Scan & 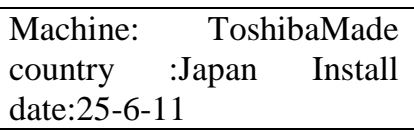 & $\begin{array}{l}\text { It is used for brain and } \\
\text { many other problem }\end{array}$ & $\begin{array}{l}\text { Restricted room, } \\
\text { entry is forbidden } \\
\text { for radiation }\end{array}$ \\
\hline & & MRI & Have but did not install & $\begin{array}{l}\text { Brain and neurological } \\
\text { purpose }\end{array}$ & $\begin{array}{l}\text { The room was } \\
\text { totally closed }\end{array}$ \\
\hline & & Endoscopy & $\begin{array}{l}\text { Machine: } \\
\text { AohuraModel:AQ- } \\
\text { 100Made country } \\
\text { :KoreaInstall date:7-02-12 }\end{array}$ & $\begin{array}{l}\text { Use for finding the } \\
\text { problem of ulcer, gastric } \\
\text { etc. }\end{array}$ & $\begin{array}{l}\text { Just saw the } \\
\text { machine }\end{array}$ \\
\hline \multirow[t]{7}{*}{2} & \multirow[t]{7}{*}{$\begin{array}{lr}\text { Dhaka } & \text { Medical } \\
\text { College } & \& \\
\text { Hospital } & \end{array}$} & X-ray & $\begin{array}{l}\text { Machine: } \\
\text { MobayashiModel: } \\
\text { Made country: } \quad \text { Korea } \\
\text { Install date: } 02.05 .2001 \\
\end{array}$ & $\begin{array}{l}\text { It is not visible, have no } \\
\text { smell and one can't sense } \\
\text { it out even it exposed with } \\
\text { high intensity }\end{array}$ & $\begin{array}{l}\text { It looked old and } \\
\text { back dated but } \\
\text { worked well }\end{array}$ \\
\hline & & Ultrasonography & $\begin{array}{l}\text { Machine: PhilipsModel: J- } \\
\text { 0046Made } \quad \text { country: } \\
\text { KoreaInstall } \\
\text { 28.03.2004 date: }\end{array}$ & $\begin{array}{l}\text { Uses for pregnant women } \\
\text { and many other diseases }\end{array}$ & $\begin{array}{l}\text { Operators were } \\
\text { helpful and operated } \\
\text { carefully }\end{array}$ \\
\hline & & CT Scan & $\begin{array}{l}\text { Machine: Scenia Made } \\
\text { country: JapanInstall date: } \\
\text { 18.04.1999 }\end{array}$ & $\begin{array}{l}\text { All structures of all } \\
\text { densities are visualized }\end{array}$ & $\begin{array}{l}\text { Enter the room and } \\
\text { see the machine for } \\
\text { short time due to } \\
\text { radiation problem }\end{array}$ \\
\hline & & MRI & $\begin{array}{l}\text { Machine: HitachiMade } \\
\text { country: JapanInstall date: } \\
\text { 07.09.2009 }\end{array}$ & $\begin{array}{l}\text { Brain and neurological } \\
\text { purpose }\end{array}$ & $\begin{array}{l}\text { Just saw the } \\
\text { machine }\end{array}$ \\
\hline & & Mammography & $\begin{array}{l}\text { Machine: SiemenMade } \\
\text { country: KoreaInstall date: } \\
07.09 .2009\end{array}$ & $\begin{array}{l}\text { Uses for finding breast } \\
\text { cancer problem }\end{array}$ & $\begin{array}{l}\text { Only saw the } \\
\text { machine }\end{array}$ \\
\hline & & ECG & $\begin{array}{l}\text { Machine: } \\
\text { SiemenModel:ECG- } \\
\text { 300GMade } \quad \text { country: } \\
\text { KoreaInstall } \\
5.12 .2013 \quad \text { date: }\end{array}$ & $\begin{array}{l}\text { Uses for finding heart } \\
\text { problem and heart beat } \\
\text { rhythm }\end{array}$ & It looked new \\
\hline & & Endoscopy & $\begin{array}{l}\text { Machine: Siemen Model: } \\
\text { CR-35Made country: Korea } \\
\text { Install date:22.7.2009 }\end{array}$ & $\begin{array}{l}\text { Uses for finding Ulcer } \\
\text { diseases }\end{array}$ & It does not look old \\
\hline \multirow[t]{4}{*}{3} & \multirow[t]{4}{*}{$\begin{array}{l}\text { Bangabandhu } \\
\text { Sheikh Mujib } \\
\text { Medical } \\
\text { University }\end{array}$} & X-ray & $\begin{array}{lr}\text { Machine: } & \text { ToshibaModel: } \\
\text { TR-0056Made } & \text { country: } \\
\text { KoreaInstall } & \text { date: } \\
02.05 .2013 & \\
\end{array}$ & $\begin{array}{l}\text { Transmitted ray passes } \\
\text { through the patient and } \\
\text { detected by x-ray film }\end{array}$ & $\begin{array}{l}\text { It looked good and } \\
\text { worked well }\end{array}$ \\
\hline & & Ultrasonography & $\begin{array}{lr}\text { Machine: } & \text { ToshibaModel: } \\
\text { DC-93Made } & \text { country: } \\
\text { JapanInstall } & \text { date: } \\
28.03 .2004 & \\
\end{array}$ & Uses for pregnancy & $\begin{array}{l}\text { Operators are very } \\
\text { carefully operating } \\
\text { the machine }\end{array}$ \\
\hline & & CT Scan & $\begin{array}{l}\text { Machine: Hitachi Made } \\
\text { country: JapanInstall date: } \\
\text { 22.05.2008 }\end{array}$ & $\begin{array}{l}\text { Image quality and } \\
\text { resolution is very high }\end{array}$ & $\begin{array}{l}\text { Discussed with the } \\
\text { operator about the } \\
\text { machine }\end{array}$ \\
\hline & & MRI & Machine: $\quad$ HitachiMade & whole body & The machine was \\
\hline
\end{tabular}




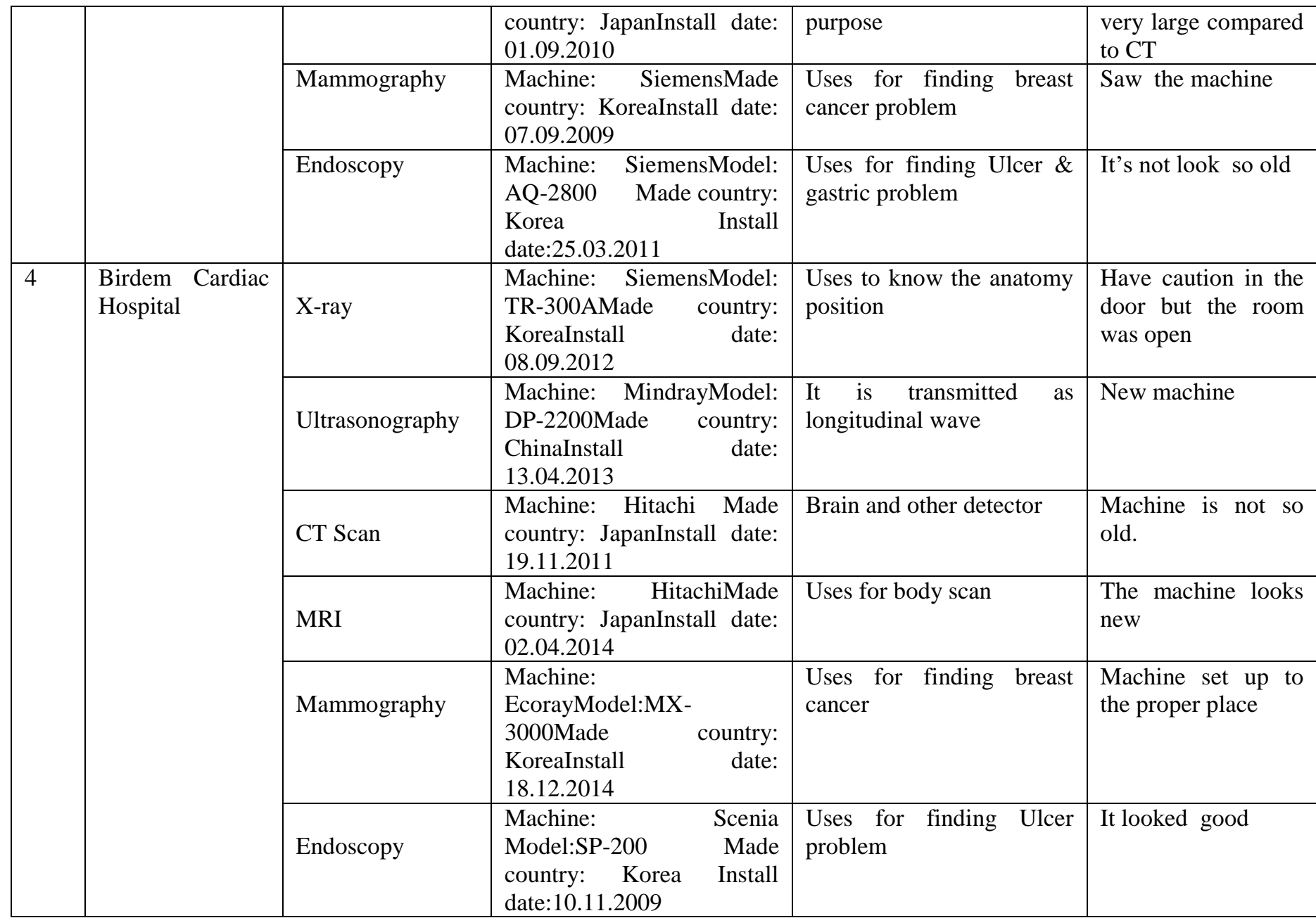

Table 4A. Equipments available and its uses in various private hospitals/clinics in Dhaka city Bangladesh

\begin{tabular}{|c|c|c|c|c|c|}
\hline No. & $\begin{array}{l}\text { Hospital/clinic } \\
\text { name }\end{array}$ & Equipment Name & $\begin{array}{l}\text { Equipment info. (Machine, } \\
\text { Machine model, made } \\
\text { country, Date of installation) }\end{array}$ & $\begin{array}{ll}\text { Application/Uses } & \text { for } \\
\text { diagnostic purposes } & \end{array}$ & Observations \\
\hline \multirow[t]{3}{*}{5} & \multirow[t]{3}{*}{$\begin{array}{l}\text { IBN-SINA } \\
\text { Medical College } \\
\text { \& Hospital }\end{array}$} & X-ray & $\begin{array}{lc}\text { Machine:CR } & \text { Wandong } \\
\text { Model:R-99ICTMade } \\
\text { country: } & \text { ChinaInstall } \\
\text { date:9.3.2009 } & \\
\end{array}$ & $\begin{array}{l}\text { Uses to know the bones } \\
\text { position }\end{array}$ & It is quite new \\
\hline & & Ultrasonography & $\begin{array}{l}\text { Machine: } \\
\text { TeknovaModel:TA- } \\
\text { 5500Made country: } \\
\text { KoreaInstall } \\
\text { date:23.04.2009 }\end{array}$ & $\begin{array}{l}\text { The velocity of sound } \\
\text { depends on the nature of } \\
\text { medium }\end{array}$ & $\begin{array}{l}\text { The doctor showed } \\
\text { us the machine and } \\
\text { its work function } \\
\text { nicely }\end{array}$ \\
\hline & & Endoscopy & $\begin{array}{l}\text { Machine: } \\
\text { SiemensModel:SP-3000 } \\
\text { Made country: KoreaInstall } \\
\text { date:03.05.06 }\end{array}$ & $\begin{array}{l}\text { Uses for gastrological } \\
\text { problem }\end{array}$ & $\begin{array}{l}\text { Machine looked old } \\
\text { so patient faces } \\
\text { some problem } \\
\text { during diagnosis }\end{array}$ \\
\hline \multirow[t]{4}{*}{6} & \multirow[t]{4}{*}{$\begin{array}{l}\text { Square Hospital } \\
\text { Limited }\end{array}$} & X-ray & $\begin{array}{l}\text { Machine: SceniaModel:YZ- } \\
\text { 500BMader country: } \\
\text { JapanInstall date:28.05.2012 }\end{array}$ & $\begin{array}{l}\text { Uses for bio medical } \\
\text { diagnostic purpose }\end{array}$ & $\begin{array}{l}\text { It looks new and the } \\
\text { operators are very } \\
\text { sincere about the test }\end{array}$ \\
\hline & & Ultrasonography & $\begin{array}{l}\text { Machine: SceniaModel:TA- } \\
\text { 5500Made country: Korea } \\
\text { Install date:09.06.2014 }\end{array}$ & $\begin{array}{l}\text { Mainly Use for pregnant } \\
\text { women to know the baby } \\
\text { position }\end{array}$ & $\begin{array}{l}\text { The operators did } \\
\text { not allow entry }\end{array}$ \\
\hline & & CT Scan & $\begin{array}{lr}\text { Machine: } & \text { ToshibaMade } \\
\text { country: } & \text { JapanInstall } \\
\text { date:03.07.2011 }\end{array}$ & $\begin{array}{l}\text { Uses for brain scan and } \\
\text { neurological problem }\end{array}$ & $\begin{array}{l}\text { Restricted room } \\
\text { entry is forbidden } \\
\text { for radiation }\end{array}$ \\
\hline & & & Machine: $\quad$ ToshibaMade & Uses for whole body scan & Saw the machine \\
\hline
\end{tabular}




\begin{tabular}{|c|c|c|c|c|c|}
\hline & & MRI & $\begin{array}{l}\text { country: JapanInstall } \\
\text { date:16.02.2013 }\end{array}$ & of the patient & outside of the room \\
\hline & & Endoscopy & $\begin{array}{l}\text { Machine: } \\
\text { TeknovaModel:AS-3000+ } \\
\text { Made country: Korea Install } \\
\text { date:02.05.09 }\end{array}$ & $\begin{array}{l}\text { Used for finding the } \\
\text { problem of ulcer, gastric } \\
\text { etc. }\end{array}$ & $\begin{array}{l}\text { Saw the work } \\
\text { function }\end{array}$ \\
\hline \multirow[t]{5}{*}{7} & \multirow[t]{5}{*}{$\begin{array}{lr}\text { Gastro } & \text { Liver } \\
\text { Hospital } & \& \\
\text { Research } & \text { center }\end{array}$} & X-ray & $\begin{array}{l}\text { Machine: PhilipsModel:I99- } \\
\text { IBTMade, country: } \\
\text { KoreaInstall date:29.05.14 }\end{array}$ & $\begin{array}{l}\text { Photographic film can be } \\
\text { made image visible }\end{array}$ & $\begin{array}{l}\text { It looked old and the } \\
\text { operators faced } \\
\text { some problem at that } \\
\text { time }\end{array}$ \\
\hline & & Ultrasonography & $\begin{array}{l}\text { Machine: } \\
\text { TeknovaModel:DP-20Made } \\
\text { country: } \quad \text { JapanInstall } \\
\text { date:4.05.14 }\end{array}$ & $\begin{array}{l}\text { The velocity of sound } \\
\text { depends on the nature of } \\
\text { medium }\end{array}$ & $\begin{array}{l}\text { The operators did } \\
\text { not allow entry }\end{array}$ \\
\hline & & CT Scan & $\begin{array}{lr}\text { Machine: } & \text { PhilipsMade } \\
\text { country: } & \text { JapanInstall } \\
\text { date:6.05.14 } & \\
\end{array}$ & $\begin{array}{l}\text { All structures of all } \\
\text { densities are visualized }\end{array}$ & $\begin{array}{l}\text { Restricted room } \\
\text { entry is forbidden } \\
\text { for radiation }\end{array}$ \\
\hline & & MRI & $\begin{array}{lr}\text { Machine: } & \text { HitachiMade } \\
\text { country: } & \text { JapanInstall } \\
\text { date:12.05.14 } & \\
\end{array}$ & $\begin{array}{l}\text { Uses for Brain and } \\
\text { neurological and whole } \\
\text { body purpose }\end{array}$ & $\begin{array}{l}\text { The room was } \\
\text { totally closed }\end{array}$ \\
\hline & & Endoscopy & $\begin{array}{l}\text { Machine: } \\
\text { AohuraModel:VMS-3500+ } \\
\text { Made country: KoreaInstall } \\
\text { date:04.05.14 }\end{array}$ & Uses for ulcer problem & $\begin{array}{l}\text { Just saw the } \\
\text { machine }\end{array}$ \\
\hline \multirow[t]{5}{*}{8} & \multirow[t]{5}{*}{$\begin{array}{l}\text { Labaid Hospital } \\
\& \quad \text { Diagnostic } \\
\text { center }\end{array}$} & X-ray & $\begin{array}{l}\text { Machine: } \\
\text { EcorayModel:F99-ICTMade } \\
\text { country: Korea Install } \\
\text { date: } 11.05 .08\end{array}$ & $\begin{array}{l}\text { Using filter can harden } \\
\text { poly energetic X-ray }\end{array}$ & $\begin{array}{l}\text { It looks old and the } \\
\text { operators faces some } \\
\text { problem at that time }\end{array}$ \\
\hline & & Ultrasonography & $\begin{array}{l}\text { Machine: } \\
\text { ChisonModel:ECM- } \\
\text { 7000Made country: } \\
\text { FranceInstall date:03.05.09 }\end{array}$ & $\begin{array}{l}\text { It has frequency more } \\
\text { than } 20 \mathrm{kHz}\end{array}$ & $\begin{array}{l}\text { The operators did } \\
\text { not allow entry }\end{array}$ \\
\hline & & CT Scan & $\begin{array}{lr}\text { Machine: } & \text { HitachiMade } \\
\text { country: } & \text { JapanInstall } \\
\text { date:08.09.10 } & \\
\end{array}$ & $\begin{array}{l}\text { Uses for neurological } \\
\text { problem }\end{array}$ & $\begin{array}{l}\text { Restricted room } \\
\text { entry is forbidden } \\
\text { for radiation }\end{array}$ \\
\hline & & MRI & $\begin{array}{lr}\text { Machine: } & \text { ToshibaMade } \\
\text { country: } & \text { JapanInstall } \\
\text { date:11.11.10 } & \\
\end{array}$ & Uses for body scan & $\begin{array}{l}\text { The room was } \\
\text { totally quiet }\end{array}$ \\
\hline & & Endoscopy & $\begin{array}{l}\text { Machine: } \\
\text { SiemensModel:SMQ-4000 } \\
\text { Made country: KoreaInstall } \\
\text { date:04.07.09 }\end{array}$ & $\begin{array}{l}\text { Uses for finding the ulcer } \\
\text { and gastric problem }\end{array}$ & $\begin{array}{l}\text { Machine was set } \\
\text { properly }\end{array}$ \\
\hline \multirow[t]{4}{*}{9} & \multirow{4}{*}{$\begin{array}{l}\text { Popular Medical } \\
\text { college Hospital } \\
\& \text { Consultation } \\
\text { Centre }\end{array}$} & X-ray & $\begin{array}{l}\text { Machine: TruipModel: DR- } \\
\text { 400Mader country: } \\
\text { ChinaInstall date:26.07.12 }\end{array}$ & $\begin{array}{l}\text { To Know the bones } \\
\text { problem }\end{array}$ & $\begin{array}{l}\text { The machine was } \\
\text { okay }\end{array}$ \\
\hline & & Ultrasonography & $\begin{array}{l}\text { Machine: ChisonModel:Ivis- } \\
\text { 60Made country: } \\
\text { ChinaInstall date:01.05.12 }\end{array}$ & $\begin{array}{l}\text { Uses to know the baby } \\
\text { position }\end{array}$ & $\begin{array}{l}\text { Got the proper } \\
\text { information from the } \\
\text { operator }\end{array}$ \\
\hline & & CT Scan & $\begin{array}{l}\text { Machine: PhilipsMade } \\
\text { country:JapanInstall } \\
\text { date:11.08.13 }\end{array}$ & $\begin{array}{l}\text { Crystal detector and } \\
\text { image reconstruction } \\
\text { occur in a digital } \\
\text { computer }\end{array}$ & $\begin{array}{l}\text { Restricted room and } \\
\text { have the caution } \\
\text { outside of the door }\end{array}$ \\
\hline & & Endoscopy & $\begin{array}{l}\text { Machine: } \\
\text { TeknovaModel:AS-200 } \\
\text { Made country: ChinaInstall } \\
\text { date: } 04.02 .13\end{array}$ & $\begin{array}{l}\text { Ulcer gastric and any } \\
\text { other purpose }\end{array}$ & Nice machine \\
\hline 10 & $\begin{array}{l}\text { Ad-din Womens } \\
\text { Medical \& } \\
\text { Hospital }\end{array}$ & X-ray & $\begin{array}{lr}\text { Machine: } & \text { EcorayModel: } \\
\text { HF525+Made } & \text { country: } \\
\text { KoreaInstall } & \\
\end{array}$ & $\begin{array}{l}\text { It can be deflected by } \\
\text { electric and magnetic field }\end{array}$ & $\begin{array}{l}\text { The operators are } \\
\text { sensitive }\end{array}$ \\
\hline
\end{tabular}




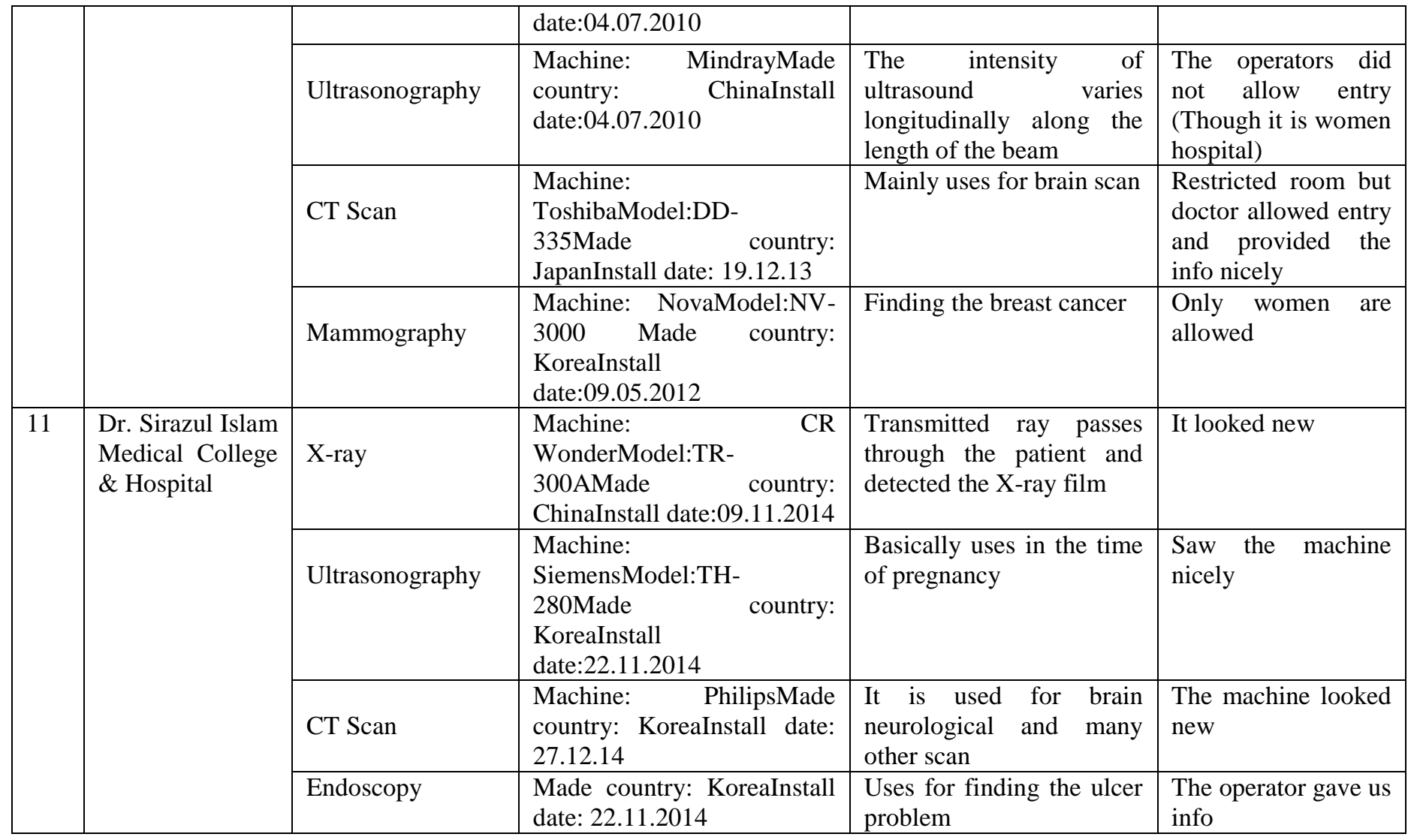

Table 4B. Equipments available and its uses in various private hospitals/clinics in Dhaka city Bangladesh

\begin{tabular}{|c|c|c|c|c|c|}
\hline No. & $\begin{array}{l}\text { Hospital/clinic } \\
\text { name }\end{array}$ & Equipment Name & $\begin{array}{l}\text { Equipment info. (Machine, } \\
\text { Machine model, made country, } \\
\text { Date of installation) }\end{array}$ & $\begin{array}{l}\text { Application/Uses } \\
\text { for diagnostic } \\
\text { purposes }\end{array}$ & Observations \\
\hline \multirow[t]{5}{*}{12} & \multirow[t]{5}{*}{$\begin{array}{l}\text { Islami Bank } \\
\text { Central } \\
\text { Hospital(Kakrail, } \\
\text { Dhaka) }\end{array}$} & X-ray $500 \mathrm{~mA}$ & $\begin{array}{l}\text { Machine: } \\
\text { HitachiModel:RadNext50Made } \\
\text { country: } \quad \text { JapanInstall } \\
\text { date:9.2.2015 }\end{array}$ & $\begin{array}{lr}\text { Diagnose } & \\
\text { fractured } & \text { bones } \\
\text { or } & \text { joint } \\
\text { dislocation } & \\
\end{array}$ & $\begin{array}{l}\text { It is very latest } \\
\text { version }\end{array}$ \\
\hline & & Ultrasonography & $\begin{array}{ll}\text { Machine: } & \\
\text { SiemenModel:AcusonX700Made } \\
\text { country: } & \text { KoreaInstall } \\
\text { date:4.01.2014 } & \end{array}$ & $\begin{array}{l}\text { Internal body } \\
\text { structures such } \\
\text { as tendons, } \\
\text { muscles, joints, } \\
\text { vessels, internal } \\
\text { organs }\end{array}$ & $\begin{array}{l}\text { New device. Last } \\
\text { year this was } \\
\text { installed. }\end{array}$ \\
\hline & & Syringe pump & $\begin{array}{lr}\text { Machine: AtomModel: } & \text { S- } \\
\text { 1235Made country: Japan } & \text { Int } \\
\text { date: } 27.8 .2015 & \end{array}$ & $\begin{array}{l}\text { Used to } \\
\text { gradually } \\
\text { administer small } \\
\text { amount of fluid } \\
\text { to a patient/ use } \\
\text { in chemical, } \\
\text { biomedical } \\
\text { research }\end{array}$ & - \\
\hline & & Haemodialysis & $\begin{array}{lll}\text { Machine: } & \text { Model: } & \text { 4008SMade } \\
\text { country: } & \text { GermanyInt date: } \\
\text { 19.6.2011 } & & \end{array}$ & $\begin{array}{lr}\text { Used r } & \text { for } \\
\text { purifying } & \text { blood } \\
\text { and remove } \\
\text { waste:involves } \\
\text { an artificial } \\
\text { kidney }\end{array}$ & $\begin{array}{l}\text { Haemodialysis } \\
\text { usage increasing }\end{array}$ \\
\hline & & ICU ventilator & $\begin{array}{l}\text { Machine: Impination 5iMade } \\
\text { country: USAInt date: } 18.9 .2013\end{array}$ & - & - \\
\hline 13 & $\begin{array}{l}\text { National Heart } \\
\text { Foundation }\end{array}$ & LaBiTec & $\begin{array}{l}\text { Made country: GermanyGmbH, } \\
\text { D-22926 }\end{array}$ & $\begin{array}{l}\text { Blood } \\
\text { coagulation and }\end{array}$ & Good condition \\
\hline
\end{tabular}




\begin{tabular}{|c|c|c|c|c|c|}
\hline & Hospital, Mirpur, & & & platelet function & \\
\hline & Dhaka & Mythic 22 & Germany 220T, AL & $\begin{array}{lr}\text { Blood: } & \text { WBC, } \\
\text { RBC, } & \text { PLT, } \\
\text { HGB, } & \text { MCV, } \\
\text { RDW, } & \text { HCT, } \\
\text { PDW } & \text { etc. } \\
\text { measuring } & \end{array}$ & $\begin{array}{ll}\text { In } & \text { good } \\
\text { condition } & \end{array}$ \\
\hline & & East Lyte Plus & Made country: USARcf3f046 & $\begin{array}{l}\text { Blood: Finding } \\
\text { the } \mathrm{Na} / \mathrm{k} / \mathrm{Cl}\end{array}$ & $\begin{array}{lr}\text { In } & \text { good } \\
\text { condition }\end{array}$ \\
\hline & & $\begin{array}{l}\text { ADVIA Centaur } \\
\text { XP }\end{array}$ & Made country: USACentour XP & $\begin{array}{l}\text { Powerful and } \\
\text { mid-sized } \\
\text { Immunoassay } \\
\text { system }\end{array}$ & $\begin{array}{ll}\text { In } & \text { good } \\
\text { condition } & \end{array}$ \\
\hline & & Celltac $\alpha$ & Made country: JapanMEK- 6400 & $\begin{array}{l}\text { Used for feline } \\
\text { and canine blood } \\
\text { samples }\end{array}$ & $\begin{array}{ll}\text { In } & \text { good } \\
\text { condition } & \end{array}$ \\
\hline & & $\mathrm{X}$-ray $500 \mathrm{~mA}$ & $\begin{array}{l}\text { Machine: HitachiModel: Fad } \\
\text { Next 50Made country: JapanInt } \\
\text { date: } 25.2 .2015\end{array}$ & \begin{tabular}{lr}
\multicolumn{2}{l}{ Used to diagnose } \\
fractured & boe \\
and & joint \\
dislocation &
\end{tabular} & $\begin{array}{ll}\text { In } & \text { good } \\
\text { condition } & \end{array}$ \\
\hline & & Ultrasonogram & 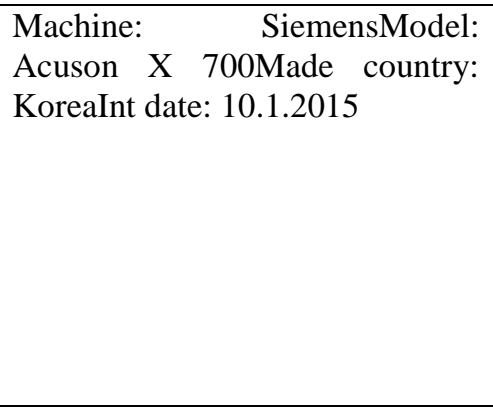 & $\begin{array}{l}\begin{array}{l}\text { Used to see } \\
\text { internal body } \\
\text { structures } \\
\text { muscles, joints, } \\
\text { vessels, liver, } \\
\text { kidney, uterus, } \\
\text { pancreas, heart, } \\
\text { lungs, prostate, } \\
\text { blood vessels } \\
\text { etc. }\end{array} \\
\end{array}$ & $\begin{array}{ll}\text { In } & \text { good } \\
\text { condition } & \end{array}$ \\
\hline 14 & $\begin{array}{l}\text { DigiLab Medical } \\
\text { Services Ltd., } \\
\text { Mirpur, Dhaka }\end{array}$ & $\begin{array}{l}\text { USG with 4D } \\
\text { Doppler }\end{array}$ & 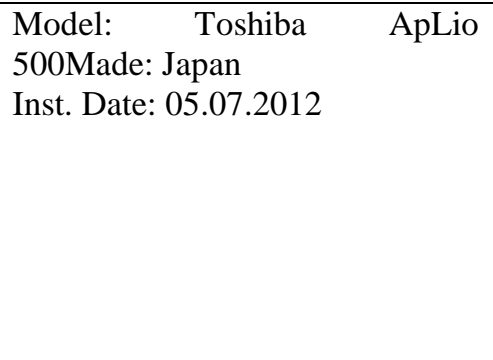 & $\begin{array}{l}\text { Suitable for use } \\
\text { in x-ray } \\
\text { fluoroscopic and } \\
\text { radiographic } \\
\text { diagnosis, used } \\
\text { in } \\
\text { gastrointestinal } \\
\text { radiographic } \\
\text { diagnosis }\end{array}$ & $\begin{array}{l}\text { Allows the } \\
\text { detection,contrast } \\
\text { and grading of } \\
\text { inflammation in } \\
\text { rhematology. }\end{array}$ \\
\hline & & $\begin{array}{l}\text { Digital X-ray with } \\
\text { computed } \\
\text { radiography }\end{array}$ & $\begin{array}{lrr}\text { DRGEM X-ray } & \text { machine } \\
\text { 500mAModel: } & \text { XD52Made } \\
\text { country: } & \text { Korealnst. } & \text { Date: } \\
17.01 .2012 & & \end{array}$ & $\begin{array}{l}\text { 1.Contrast- } \\
\text { enhanced } \\
\text { ultrasound, } \\
\text { 2)Contrast } \\
\text { quantification } \\
\text { 3)Realtime } \\
\text { elastrography 4) } \\
\text { Shear wave } \\
\text { elastrography 5) } \\
\text { Propagation } \\
\text { mapping }\end{array}$ & $\begin{array}{l}\text { preferable for } \\
\text { radiography such } \\
\text { as general body, } \\
\text { beam filtration } \\
\text { and chest }\end{array}$ \\
\hline & & $\begin{array}{ll}\text { Echo } & \text { Color } \\
\text { Doppler, EEG, } & \text { EET, Video } \\
\text { EET, Endoscope, } \\
\text { Mammography, } \\
\text { OPG, Dental X- } \\
\text { ray, Multislice CT }\end{array}$ & 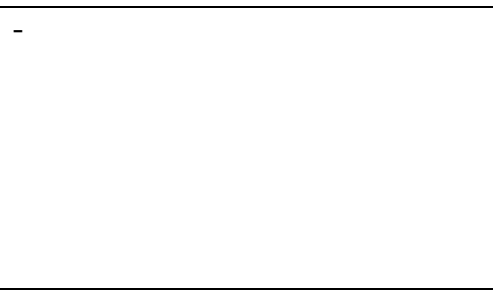 & & - \\
\hline 15 & $\begin{array}{l}\text { Islamia General } \\
\text { Hospital, North } \\
\text { Jatrabari, Dhaka }\end{array}$ & UCG machine & 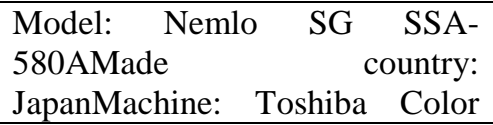 & $\begin{array}{l}\text { For imaging } \\
\text { inside the body }\end{array}$ & Digital \\
\hline
\end{tabular}




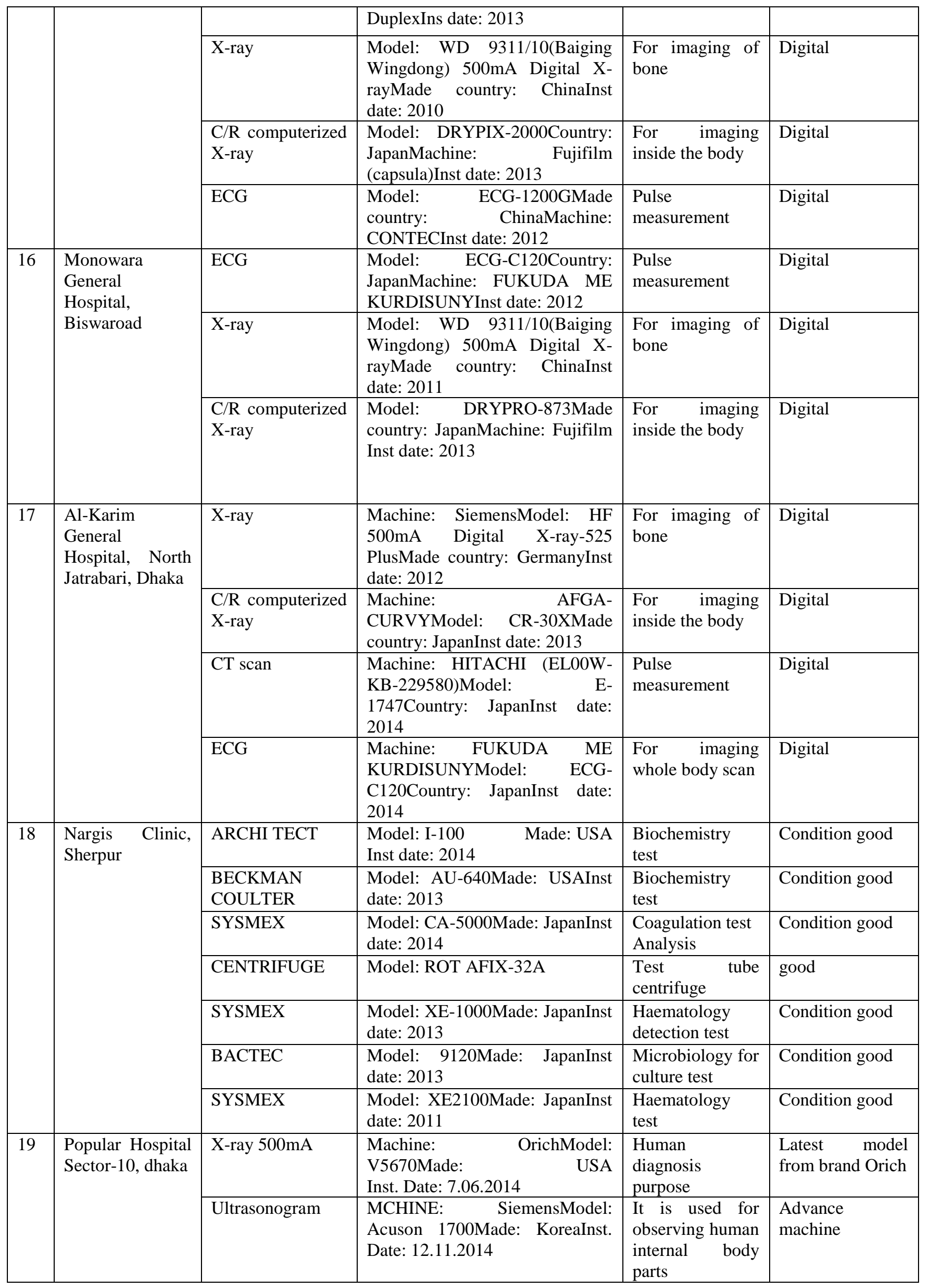




\begin{tabular}{|c|c|c|c|c|c|}
\hline & & MRI & $\begin{array}{lr}\text { Machine: } & \text { PhilipsModel: } \\
\text { 3.OTMade: } & \text { NetherlandsInst. } \\
\text { Date: 07.09.2013 } & \\
\end{array}$ & $\begin{array}{l}\text { For diagnosis } \\
\text { purpose }\end{array}$ & $\begin{array}{l}\text { Most costly } \\
\text { equipment in the } \\
\text { hospital }\end{array}$ \\
\hline & & ICU ventilator & $\begin{array}{l}\text { Machne: } \quad \text { ImpinationModel: } \\
\text { 5iInst. Date: } 18.09 .2013\end{array}$ & $\begin{array}{l}\text { This is to blow } \\
\text { air with mixture } \\
\text { of gases into the } \\
\text { lungs }\end{array}$ & $\begin{array}{l}\text { This is clean and } \\
\text { protected. }\end{array}$ \\
\hline \multirow[t]{5}{*}{20} & \multirow[t]{5}{*}{$\begin{array}{l}\text { Ayesha } \\
\text { Memorial, } \\
\text { Mokakhali }\end{array}$} & $\begin{array}{l}\text { X-ray machine } \\
500 \mathrm{~mA}\end{array}$ & $\begin{array}{l}\text { Brand: JPI HealthcarModel: } \\
\text { DRE-180Made: JapanInst date: } \\
\text { 2013e }\end{array}$ & $\begin{array}{l}\text { Bone } \\
\text { measurement }\end{array}$ & Good condition \\
\hline & & $\begin{array}{ll}\text { Full digital } & 12 \\
\text { channel ECG } & \end{array}$ & $\begin{array}{l}\text { Model: EC-12TMade: RussiaInst } \\
\text { date: } 2014\end{array}$ & $\begin{array}{l}\text { Heart rate } \\
\text { analyzer, systolic } \\
\text { and diastolic } \\
\text { measure }\end{array}$ & Good condition \\
\hline & & MRI & 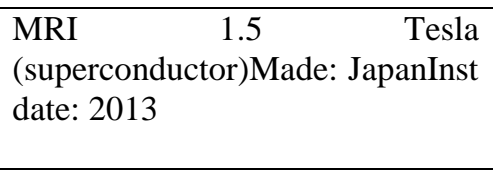 & $\begin{array}{lr}\text { Brain, } & \text { neck, } \\
\text { head, } & \text { glands, } \\
\text { kidney, liver, } & \text { leg, foot etc. } \\
\end{array}$ & Good condition \\
\hline & & Analyzer & $\begin{array}{lr}\text { Machine: } & \text { CormyModel: } \\
\text { Multi+Made: } & \text { PolandInst date: } \\
2012 & \end{array}$ & $\begin{array}{l}\text { Blood analyzer, } \\
\text { blood refine }\end{array}$ & Good condition \\
\hline & & OT table & $\begin{array}{ll}\text { Machine: } & \text { LEWINMade: } \\
\text { JapanInst date: } 2014\end{array}$ & $\begin{array}{l}\text { Hydraulic and } \\
\text { Electric OT table }\end{array}$ & Good condition \\
\hline \multirow[t]{3}{*}{21} & \multirow[t]{3}{*}{$\begin{array}{l}\text { Khidmah } \\
\text { Hospital, } \\
\text { Biswaorad, } \\
\text { Dhaka }\end{array}$} & $\begin{array}{l}\text { X-ray } \\
500 \mathrm{~mA}\end{array}$ & $\begin{array}{l}\text { Machine: } \quad \text { SiemensModel: } \\
\text { Radnou+55Made: GermanyInst. } \\
\text { Date: } 3.3 .2016\end{array}$ & $\begin{array}{l}\text { Used to diagnose } \\
\text { joint dislocation } \\
\text { and fractures } \\
\text { bones }\end{array}$ & Latest machine \\
\hline & & Ultra sonogram & $\begin{array}{l}\text { Machine: } \quad \text { Siemens } 4 \mathrm{D} \\
\text { colorModel: Acuson X-500Inst. } \\
\text { Date: } 04.05 .2012\end{array}$ & $\begin{array}{l}\text { It is used to see } \\
\text { internal body } \\
\text { structures such } \\
\text { as tendon, } \\
\text { muscles, joints, } \\
\text { vessels, internal } \\
\text { organs }\end{array}$ & New device \\
\hline & & Syringe pump & \begin{tabular}{llr} 
Machine: & \multicolumn{2}{r}{ AtomModel: } \\
1235Made: & JapanInst. & Date: \\
27.10.2015 & &
\end{tabular} & $\begin{array}{l}\text { Used for } \\
\text { administering } \\
\text { small amount of } \\
\text { fluid into patient, } \\
\text { used for } \\
\text { chemical and } \\
\text { biomedical } \\
\text { research }\end{array}$ & New device \\
\hline \multirow[t]{5}{*}{22} & \multirow{5}{*}{$\begin{array}{l}\text { Badda General } \\
\text { Hospital, Uttar } \\
\text { Badda, } \\
\text { Gulshan1,Dhaka }\end{array}$} & Incubator & Model: jouan 36 & $\begin{array}{l}\text { Sterilization, } \\
\text { microbiological } \\
\text { test }\end{array}$ & New \\
\hline & & Autoclave & Made: China & $\begin{array}{l}\text { Sterilization for } \\
\text { glass tube, test } \\
\text { tube and others }\end{array}$ & New \\
\hline & & Sysmax & $\begin{array}{l}\text { Model: KX21Made: JapanInst. } \\
\text { Date: } 2012\end{array}$ & $\begin{array}{l}\text { Hematological } \\
\text { purpose; detect } \\
\text { for CBC, ADL, } \\
\text { blood platelets, } \\
\text { dengue fever }\end{array}$ & New \\
\hline & & Micro-ER & $\begin{array}{l}\text { Model: ER-500Made: China } \\
\text { Inst. Date: } 2012\end{array}$ & $\begin{array}{l}\text { Hematological } \\
\text { purpose used for } \\
\text { hormonal assay, } \\
\text { detect hormone } \\
\text { problem }\end{array}$ & New \\
\hline & & $\begin{array}{l}\text { Evolution } \\
\text { machine }\end{array}$ & $\begin{array}{l}\text { Model: } 300 \quad \text { EvolutionMade: } \\
\text { Japan }\end{array}$ & $\begin{array}{l}\text { All biological } \\
\text { test, } \mathrm{S} \text {, bilirubin, }\end{array}$ & Old \\
\hline
\end{tabular}




\begin{tabular}{|c|c|c|c|c|c|}
\hline & & & & $\mathrm{RB}, \mathrm{SFBs}$ & \\
\hline & & $\begin{array}{l}\text { Centrifugal } \\
\text { machine }\end{array}$ & Model: SB-100Made: Japan & SPPR & Old \\
\hline & & Hot air oven & Made: Japan & $\begin{array}{lr}\text { Protect } & \text { all } \\
\text { equipment } & \text { in } \\
\text { diagnosis } & \\
\end{array}$ & Old \\
\hline & & Ultrasono & Model: Logiq-2200Made: China & $\begin{array}{l}\text { Mostly used for } \\
\text { liver, stomach, } \\
\text { kidney problem }\end{array}$ & Old \\
\hline & & ECG & $\begin{array}{l}\text { Model: Cardiofax } \text { ECiMade: } \\
\text { Japan }\end{array}$ & $\begin{array}{l}\text { Used for chest } \\
\text { problem }\end{array}$ & Old \\
\hline & & VLTROS & Model: ECiMade: England & Unknown & Old \\
\hline & & CT scanner & Siemens CT & $\begin{array}{l}\text { Mostly used for } \\
\text { brain scanning }\end{array}$ & Old \\
\hline & & X-ray & $\begin{array}{l}\text { Model: CR-30-X Arfa Made: } \\
\text { Japan }\end{array}$ & $\begin{array}{l}\text { Used for chest } \\
\text { and other parts } \\
\text { of body checking }\end{array}$ & Old \\
\hline & & $\begin{array}{l}\text { Nebulizing } \\
\text { machine }\end{array}$ & Made: China & $\begin{array}{l}\text { breathing } \\
\text { treatment }\end{array}$ & Old \\
\hline & & Evolis & Model: Twin plus & Unknown & Old \\
\hline & & Dimension & Model: Rx2 max & Unknown & Old \\
\hline & & $\begin{array}{l}\text { VESMATIC } \\
\text { CUBE }\end{array}$ & $\begin{array}{ll}\text { Model: } & \text { UnknownMade: } \\
\text { Germany } & \\
\end{array}$ & Unknown & Old \\
\hline 23 & $\begin{array}{l}\text { Pancare Hospital } \\
\text { Ltd. } \\
\text { Dhanmondi R/A, } \\
\text { Dhaka }\end{array}$ & $\begin{array}{l}\text { Magnamed } \\
\text { (NICU) }\end{array}$ & $\begin{array}{l}\text { Model: GT-2000Made: Japan, } \\
\text { Inst. Date: } 2014\end{array}$ & $\begin{array}{l}\text { Used as a } \\
\text { ventilator in } \\
\text { NICU as infant } \\
\text { warmer }\end{array}$ & Good condition \\
\hline & & Incubator (infant) & $\begin{array}{l}\text { Model: CE0197-B3000Made: } \\
\text { GermanyInst. Date: } 2009\end{array}$ & $\begin{array}{l}\text { Incubator as life } \\
\text { support } \\
\text { infant }\end{array}$ & Good condition \\
\hline & & Ventilator (PICU) & $\begin{array}{l}\text { Model: Takaoka-6060Made: } \\
\text { Brazil, Int. date2008 }\end{array}$ & Ventilator & Good condition \\
\hline & & Pulse Oximetal & $\begin{array}{l}\text { Model: NT2A, Made: } \\
\text { Inst. Date: } 2009\end{array}$ & $\begin{array}{l}\text { Measure the } \\
\text { pulse rate of the } \\
\text { infant patient }\end{array}$ & Good condition \\
\hline 24 & $\begin{array}{lr}\text { Aryan } & \text { General } \\
\text { Hospital } & \text { and }\end{array}$ & X-ray Machine & $\begin{array}{l}\text { Model: Lumimos.RSMade: } \\
\text { Germany, Inst. Date: } 2005\end{array}$ & $\begin{array}{l}\text { Takes images of } \\
\text { specific bones }\end{array}$ & Digital \\
\hline & $\begin{array}{l}\text { Diagnostic } \\
\text { center, Khilkhet }\end{array}$ & Dental machine & $\begin{array}{l}\text { Model: } \quad \text { CramexaMade: } \\
\text { GermanyInst. Date: } 2006\end{array}$ & $\begin{array}{l}\text { Check teeth by } \\
\text { the machine }\end{array}$ & Digital \\
\hline & & Sysmex & $\begin{array}{l}\text { Model: XT2000CMade: Finland, } \\
\text { Inst. Date: } 2010\end{array}$ & $\begin{array}{l}\text { Pathology } \\
\text { purpose }\end{array}$ & Digital \\
\hline & & VESMATIC cube & $\begin{array}{l}\text { Model: 80Made: GermanyInst. } \\
\text { Date: } 2010\end{array}$ & $\begin{array}{l}\text { Use for } \\
\text { Ametological } \\
\text { purpose }\end{array}$ & Digital \\
\hline & & ECG & $\begin{array}{l}\text { Model: Toshiba- ISTYLIMade: } \\
\text { Japan, Inst. Date: } 2005\end{array}$ & $\begin{array}{l}\text { Used for dental } \\
\text { purpose }\end{array}$ & Digital \\
\hline & & CRAtex & $\begin{array}{l}\text { Model: ExcelMade: JapanInst. } \\
\text { Date: } 2006\end{array}$ & To pulse check & Digital \\
\hline 25 & $\begin{array}{l}\text { Dogma } \\
\text { Diagnostic } \\
\text { center, Uttar } \\
\text { Badda }\end{array}$ & X-ray & $\begin{array}{l}\text { Model: MAINSMade: JapanInst. } \\
\text { Date: } 2008\end{array}$ & $\begin{array}{lr}\begin{array}{l}\text { Used } \\
\text { checking }\end{array} & \begin{array}{r}\text { for } \\
\text { the }\end{array} \\
\text { body, } & \text { teeth } \\
\text { purpose } & \\
\end{array}$ & Digital \\
\hline & & Dental & $\begin{array}{l}\text { Model: } \quad \text { Panpas-EMade: } \\
\text { JapanInst. Date: } 2009\end{array}$ & $\begin{array}{l}\text { To absorb teeth } \\
\text { purpose }\end{array}$ & Digital \\
\hline & & FCR & $\begin{array}{l}\text { Model: } \quad \text { CAPSULAMade: } \\
\text { JapanInst. Date: } 2008\end{array}$ & $\mathrm{X}$-ray purpose & Digital \\
\hline & & controller & Model: $\quad$ FUJIFILMMade: & To absorb X-ray & Digital \\
\hline
\end{tabular}




\begin{tabular}{|c|c|c|c|c|c|}
\hline & & machine & JapanInst. Date: 2008 & machine control & \\
\hline & & Pathology printer & $\begin{array}{l}\text { Model: DRY PI X 2000Made: } \\
\text { GermanyInst. Date: } 2008\end{array}$ & Used for printing & Digital \\
\hline & & CRALEX & $\begin{array}{l}\text { Model: ExcelMade: JapanInst. } \\
\text { Date: } 2008\end{array}$ & Dental purpose & Digital \\
\hline & & ECG & $\begin{array}{l}\text { Model: Toshiba-ISTYLIMade: } \\
\text { JapanInst. Date: } 2007\end{array}$ & To check pulse & Digital \\
\hline & & NIHONKOHDED & $\begin{array}{lrr}\text { Model: } & \text { Cardeofax } & \text { SMade: } \\
\text { JapanInst. Date: } 2007 & \\
\end{array}$ & $\begin{array}{l}\text { Attached on } \\
\text { ECG monitor }\end{array}$ & Digital \\
\hline 26 & $\begin{array}{l}\text { United Hospital, } \\
\text { Gulshan2, Dhaka }\end{array}$ & ARCHITECT & $\begin{array}{l}\text { Model: } \quad \text { ARCHITECT } \\
\text { 1000SRMade: } \\
05.01 .2015\end{array}$ & $\begin{array}{l}\text { Biochemistry } \\
\text { test analyzer. } \\
\text { e,g, URTA, } \\
\text { SGPT, SGOT, } \\
\text { etc. }\end{array}$ & - \\
\hline & & SYSMEX & $\begin{array}{l}\text { Model: CA500Made: JapanInst. } \\
\text { Date: } 15.03 .2015\end{array}$ & $\begin{array}{ll}\text { Coagulation } & \text { test } \\
\text { analyzer. } & \text { e.g. } \\
\text { PT, etc. } & \end{array}$ & - \\
\hline & & SYSMEX & $\begin{array}{l}\text { Model: XE1000Made: JapanInst. } \\
\text { Date: } 03.04 .2015\end{array}$ & $\begin{array}{l}\text { Haematology } \\
\text { test e.g. CBC }\end{array}$ & - \\
\hline & & ARCHITECT & $\begin{array}{lll}\text { Model: } & \text { CI8200Made: } & \text { USA } \\
\text { Inst. Date: } & 01.04 .2015 & \end{array}$ & $\begin{array}{l}\text { Biochemistry } \\
\text { test analyzer e.g. } \\
\text { SGPT, SGOT } \\
\text { etc. }\end{array}$ & - \\
\hline & & $\begin{array}{l}\text { Centrifuge } \\
\text { machine }\end{array}$ & $\begin{array}{l}\text { Model: ROTAFIX 32AMade: } \\
\text { ChinaInst. Date: } 02.02 .2014\end{array}$ & $\begin{array}{l}\text { Used for test } \\
\text { tube centrifuge }\end{array}$ & - \\
\hline & & SYSMEX & $\begin{array}{lcc}\text { Model: } & \text { XE } & \text { 2100Made: } \\
\text { JapanInst. Date:10.12.2011 }\end{array}$ & $\begin{array}{l}\text { Haematology } \\
\text { test e.g. CBC, } \\
\text { ESR, CE }\end{array}$ & - \\
\hline & & BACTEC & $\begin{array}{l}\text { Model: 9120Made: JapanInst. } \\
\text { Date:01.01.2014 }\end{array}$ & $\begin{array}{l}\text { Microbiology for } \\
\text { blood culture test }\end{array}$ & - \\
\hline & & D-10 & $\begin{array}{l}\text { Model: D-10Made: JapanInst. } \\
\text { Date: 01.02.2015 }\end{array}$ & $\begin{array}{l}\text { Biochemistry } \\
\text { test specially for } \\
\text { HBAIC }\end{array}$ & - \\
\hline & & $\begin{array}{l}\text { X-ray } \\
500 \mathrm{~mA}\end{array}$ & $\begin{array}{l}\text { Brand: JPI HealthCareModel: } \\
\text { DRE 140Made: KoreaInst. Date: } \\
2014\end{array}$ & $\begin{array}{l}\text { Bone } \\
\text { measurement }\end{array}$ & Good condition \\
\hline & & ECG & $\begin{array}{l}\text { Model: EC12TMade: RussiaInst. } \\
\text { Date: } 2013\end{array}$ & $\begin{array}{ll}\begin{array}{l}\text { Heart } \\
\text { analyzer, }\end{array} & \text { rate } \\
\text { Systolic } & \text { and } \\
\text { Diastolic } & \\
\text { measure } & \\
\end{array}$ & Full digital good \\
\hline & & Ultrasonography & $\begin{array}{l}\text { Machine: AlpinonModel: E } \\
\text { cube-15Made: USAInst. Date: } \\
2012\end{array}$ & $\begin{array}{l}\text { Golbladder, } \\
\text { thyroid, kidney, } \\
\text { heart }\end{array}$ & Good \\
\hline & & OT table & $\begin{array}{l}\text { Machine: } \quad \text { LEWINMade: } \\
\text { USAInst. Date: } 2010\end{array}$ & $\begin{array}{l}\text { Electric, } \\
\text { hydrolytic OT } \\
\text { table }\end{array}$ & $\begin{array}{ll}\text { Very } & \text { good } \\
\text { condition } & \end{array}$ \\
\hline 27 & $\begin{array}{l}\text { Apollo Hospital, } \\
\text { Dhaka }\end{array}$ & $\begin{array}{l}\text { Has a lot of good } \\
\text { equipments }\end{array}$ & - & - & $\begin{array}{ll}\text { In } & \text { good } \\
\text { condition } & \end{array}$ \\
\hline 28 & $\begin{array}{l}\text { Labaid Hospital, } \\
\text { Gulshan2, Dhaka }\end{array}$ & Ultrasonogram & $\begin{array}{l}\text { Brand: SiemensModel: } \\
\text { X7cuson } \\
\text { 5.07.2014: Kade: KoreaInst. Date: }\end{array}$ & $\begin{array}{l}\text { Used to observe } \\
\text { human body } \\
\text { parts }\end{array}$ & $\begin{array}{l}\text { Advanced } \\
\text { machine }\end{array}$ \\
\hline & & MRI & $\begin{array}{l}\text { Brand: PhilipsModel: 3.0tMade: } \\
\text { USAInst. Date: 07.06.2015 }\end{array}$ & $\begin{array}{l}\text { It is used to blow } \\
\text { air on a mixture } \\
\text { of gases into the } \\
\text { lungs }\end{array}$ & Costly equipment \\
\hline & & X-ray $500 \mathrm{~mA}$ & $\begin{array}{lcr}\text { Machine: } & \text { OrichModel: } & \text { V } \\
\text { 5670Made: USAInst. Date: } 2013\end{array}$ & $\begin{array}{l}\text { Human } \\
\text { diagnosis } \\
\text { purpose }\end{array}$ & $\begin{array}{l}\text { Latest } \\
\text { model }\end{array}$ \\
\hline
\end{tabular}




\begin{tabular}{|c|c|c|c|c|c|}
\hline & & CT scan & $\begin{array}{l}\text { Model: I-1000SRMade: USA, } \\
\text { Inst. Date: } 2014\end{array}$ & $\begin{array}{l}\text { Human full body } \\
\text { test purpose }\end{array}$ & Large machine \\
\hline & & SYSMEX & Model: X5-1000Made: Japan & $\begin{array}{l}\text { Hematology } \\
\text { detection test }\end{array}$ & \\
\hline \multirow[t]{12}{*}{29} & \multirow{12}{*}{$\begin{array}{l}\text { IBN-SINA } \\
\text { diagnostic and } \\
\text { Consultation } \\
\text { center, Uttar } \\
\text { Badda }\end{array}$} & X-ray machine & $\begin{array}{l}\text { Model: LUMINOS.RSMade: } \\
\text { GermanyInst. Date: } 11.2 .2010\end{array}$ & $\begin{array}{l}\text { Used to check } \\
\text { body and bone }\end{array}$ & New and digital \\
\hline & & CT scan & $\begin{array}{l}\text { Model: SOMAT6M SpirtMade: } \\
\text { GermanyInst. Date: } 21.1 .2013\end{array}$ & $\begin{array}{l}\text { Used in brain } \\
\text { and check whole } \\
\text { body }\end{array}$ & New and digital \\
\hline & & $\begin{array}{l}\text { DENTAL } \\
\text { machine }\end{array}$ & $\begin{array}{l}\text { Model: CRANEX-AMade: } \\
\text { FinlandInst. Date: } 2.10 .2012\end{array}$ & $\begin{array}{l}\text { Used in teeth test } \\
\text { purposes }\end{array}$ & Digital \\
\hline & & SYSMEX & $\begin{array}{l}\text { Model: XT2000CMade: Japan, } \\
\text { Inst. Date: } 2013\end{array}$ & $\begin{array}{l}\text { It is used in } \\
\text { amatological } \\
\text { diagnostic } \\
\text { purposes }\end{array}$ & Digital \\
\hline & & $\begin{array}{l}\text { VESMATIC } \\
\text { CUBE }\end{array}$ & $\begin{array}{l}\text { Model: 80Made: GermanyInst. } \\
\text { Date: } 2.10 .2010\end{array}$ & - & - \\
\hline & & BD BACTEC & $\begin{array}{l}\text { Model: FX 40, Made: USA, Inst. } \\
\text { Date: } 20.01 .2013\end{array}$ & $\begin{array}{l}\text { It is used in } \\
\text { blood culture } \\
\text { sensitive test }\end{array}$ & New and digital \\
\hline & & SIFT & $\begin{array}{lcc}\text { Model: } & \text { MN } & \text { 090Made: } \\
\text { TurkeyInst. Date: } & \text { 20.01.2013 }\end{array}$ & - & Digital \\
\hline & & Dimension & $\begin{array}{l}\text { Model: } \quad \text { RXL } \quad \text { MAXMade: } \\
\text { GermanyInst. Date: }\end{array}$ & $\begin{array}{l}\text { Biochemistry } \\
\text { test for } \\
\text { diagnostic } \\
\text { purposes }\end{array}$ & Digital \\
\hline & & VICTROS & $\begin{array}{l}\text { Model: ECIMade: UKInst. Date: } \\
\text { 10.12.2009 }\end{array}$ & $\begin{array}{l}\text { Used in } \\
\text { imological test }\end{array}$ & Digital \\
\hline & & IMMULITE & $\begin{array}{l}\text { Model: Immulite 2000Made: } \\
\text { GermanyInst. Date: } 27.07 .2010\end{array}$ & $\begin{array}{l}\text { Used in } \\
\text { imological test }\end{array}$ & Digital \\
\hline & & DADEBEHRING & $\begin{array}{l}\text { Model: BN PROSPECEMade: } \\
\text { GermanyInst. Date: } 28.12 .2013\end{array}$ & $\begin{array}{l}\text { Used in } \\
\text { serological test }\end{array}$ & Digital \\
\hline & & EVOLIS & 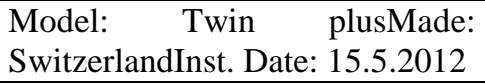 & $\begin{array}{l}\text { Used in Alaiga } \\
\text { various panel }\end{array}$ & New and digital \\
\hline
\end{tabular}

Table 5 shows the major equipments availability on 11 major hospitals and clinics in Dhaka. It is found that x-ray, ultrasound and CT are present in all 11 major hospitals in Dhaka. While endoscopy is present in all but one hospital. Also, PET is not available in any of the hospitals. Mammography is present in 6 hospitals, while MRI is present in 7 hospitals amongst which one of them is not installed yet. EEG is available in 3 hospitals amongst which 2 are public, and ECG is available in 7 hospitals amongst the 11 major hospitals/clinics in Dhaka. All the major hospitals have X-ray and ultrasound machine and some hospital do not have CT or MRI machine. The percentage of X-ray and Ultrasound machine is $100 \%$ and another CT or MRI machine is $90 \%$ available on our researched hospital in Dhaka city.

Table 6 shows the costs of usage of various equipments in public and private hospitals in Dhaka. Mostly the poor patients go to the public hospitals for treatment and usage of the various medical equipments as it is much cheaper there. There is a huge line and rush in these public hospitals. Patients have accessibility to these equipments as the doctors prescribe various tests for diagnosis and treatment purposes. Hospital, clinic and diagnostic centers show off their equipment services in banner and leaflets. The number of equipments in Dhaka city are not enough for the diagnosis and treatment of the patients who come also from other parts of the country to the capital for better treatment. Most of the well-known doctors try to stay in capital and there are more treatment equipments and facilities in Dhaka than in other parts of Bangladesh. Medical equipments costs also can be reduced for better access for diagnosis and treatment purposes.

In this research, some public (government) and private (nongovernment) hospitals/clinics of Bangladesh were visited. Some data about medical imaging instrument were taken. There are some common instruments like x-ray, ultrasound machine, endoscopy, computed tomography machine. But MRI, linac accelerator, mammography machine are not available everywhere. It was also found that most of the instruments are made by Japan, Korea, China and others countries. Bangladesh only imports that machine. So it can be said that Bangladesh is not so updated with medical imaging. In this research we saw the condition of public and private hospitals/clinics in Dhaka in terms of medical imaging instruments condition. 
Table 5: Table 5. Summary of frequently used instruments available in various major 11 public and private medical hospitals/clinics in Dhaka city. "P" stands for instrument present in the hospital/clinic, while "A" means not available.

\begin{tabular}{|c|c|c|c|c|c|c|c|c|c|}
\hline Name of hospital & X-ray & $\begin{array}{l}\text { Ultra } \\
\text { sound }\end{array}$ & CT & MRI & EEG & ECG & Endoscopy & Mamography & PET \\
\hline S.S.M.C & $\mathrm{P}$ & $\mathrm{P}$ & $\mathrm{P}$ & $\mathrm{P}$ (not installed) & $\mathrm{P}$ & $\mathrm{P}$ & $\mathrm{P}$ & A & $\mathrm{A}$ \\
\hline $\mathrm{DMCH}$ & $\mathrm{P}$ & $\mathrm{P}$ & $\mathrm{P}$ & $\mathrm{P}$ & $\mathrm{P}$ & $\mathrm{P}$ & $\mathrm{P}$ & $\mathrm{P}$ & $\mathrm{A}$ \\
\hline Bangabandhu MC & $\mathrm{P}$ & $\mathrm{P}$ & $\mathrm{P}$ & $\mathrm{P}$ & $\mathrm{A}$ & $\mathrm{P}$ & $\mathrm{P}$ & $\mathrm{P}$ & $\mathrm{A}$ \\
\hline Birdem & $\mathrm{P}$ & $\mathrm{P}$ & $\mathrm{P}$ & $\mathrm{P}$ & A & $\mathrm{P}$ & $\mathrm{P}$ & $\mathrm{P}$ & $\mathrm{A}$ \\
\hline Ibn Sina & $\mathrm{P}$ & $\mathrm{P}$ & $\mathrm{P}$ & $\mathrm{A}$ & $\mathrm{A}$ & $\mathrm{A}$ & $\mathrm{P}$ & $\mathrm{A}$ & $\mathrm{A}$ \\
\hline Square & $\mathrm{P}$ & $\mathrm{P}$ & $\mathrm{P}$ & $\mathrm{P}$ & $\mathrm{A}$ & $\mathrm{P}$ & $\mathrm{P}$ & $\mathrm{A}$ & $\mathrm{A}$ \\
\hline Gastro Liver Hospital & $\mathrm{P}$ & $\mathrm{P}$ & $\mathrm{P}$ & $\mathrm{P}$ & $\mathrm{A}$ & A & $\mathrm{P}$ & $\mathrm{A}$ & $\mathrm{A}$ \\
\hline Labaid & $\mathrm{P}$ & $\mathrm{P}$ & $\mathrm{P}$ & $\mathrm{P}$ & $\mathrm{P}$ & $\mathrm{P}$ & $\mathrm{P}$ & $\mathrm{P}$ & $\mathrm{A}$ \\
\hline Popular & $\mathrm{P}$ & $\mathrm{P}$ & $\mathrm{P}$ & $\mathrm{A}$ & $\mathrm{A}$ & $\mathrm{P}$ & $\mathrm{P}$ & $\mathrm{A}$ & $\mathrm{A}$ \\
\hline Ad-din & $\mathrm{P}$ & $\mathrm{P}$ & $\mathrm{P}$ & $\mathrm{A}$ & $\mathrm{A}$ & $\mathrm{A}$ & $\mathrm{A}$ & $\mathrm{P}$ & $\mathrm{A}$ \\
\hline Dr Sirajul Islam & $\mathrm{P}$ & $\mathrm{P}$ & $\mathrm{P}$ & $\mathrm{A}$ & $\mathrm{A}$ & $\mathrm{A}$ & $\mathrm{P}$ & $\mathrm{A}$ & $\mathrm{A}$ \\
\hline
\end{tabular}

Table 6. Summary of frequently used instruments cost for patients in public and private hospitals/clinics/diagnostic centers in Dhaka city and in outside village (rural area).

\begin{tabular}{|c|c|c|c|}
\hline Instrument Name & $\begin{array}{l}\text { Cost } \quad \text { in } \\
\text { Hospital(Dhaka City) in Taka }\end{array}$ & $\begin{array}{l}\text { Cost in } \quad \text { Private } \\
\text { Hospital(Dhaka City) in taka }\end{array}$ & $\begin{array}{l}\text { Cost in Village } \\
\text { Hospital in Taka }\end{array}$ \\
\hline X-ray & $100-400$ & $400-1800$ & $400-1200$ \\
\hline Ultrasonography & $100-500$ & $400-1500$ & $420-1000$ \\
\hline ECG & 100 & $400-800$ & $500-600$ \\
\hline CT Scan & $2000-2500$ & $4000-5500$ & $\begin{array}{l}\text { Rare in village but } \\
\text { some costlier from } \\
\text { city }\end{array}$ \\
\hline MRI & $2000-3000$ & $5000-7000$ & Didn't see in village \\
\hline
\end{tabular}

\section{DISCUSSION}

Medical imaging was first started in $17^{\text {th }}$ century. X-ray machine was first published in 1895 and now in 2016 it is updated continuously. There are many instruments and technologies which are not used in our country. Some technology which are not yet used in Bangladesh like CAVEman (image), Corneal topography, Simultaneous Algebraic Reconstruction Technique Preclinical imaging, Algebraic Reconstruction Technique, Altropane, Ambivu 3D Workstation,Amorphous Selenium Flat Panel Detectors Amplitude of low frequency fluctuations Analysis of Functional NeuroImages, Anthropometry, Nanotomography, National Coalition for Quality Diagnostic Imaging Services, Neutron stimulated emission computed tomography, Noncontact thermography. Machlett Laboratories Magnetic field imaging, Magnetic resonance elastography, Magnetogastrography, Medcyclopaedia Medical animation, Medical image computing, Medical Image Sharing, Medical ultrasound Medipix MICAD, MicroDicom ,MicroDose, Molecular imaging, Multiscale motion map and many others. They all are modern biomedical instruments and technology.

In this research, we discussed about biomedical imaging and recent condition of biomedical imaging instruments and their usage in Bangladesh. Medical imagining is very important sector all over the world. In Bangladesh this sector is not so popular. Many kinds of medical instruments were found like x-ray, ultrasound machine, CT, MRI, ECG, EEG, mammography etc. But they all are not available. In public (government) hospitals, there is lack of medical imaging instruments. Private (non-government) hospital keep more medical instruments. But is very high cost in non-government hospital.

Radiation Effect from medical equipments and Protection Background radiation refers to the ionizing radiation from high energy particles or rays that we are unavoidably exposed to in our daily lives, which gives each of us a small but continuous dose of ionizing radiation. All persons are weakly radioactive due to the presence of radioactive elements in our bodies (e.g. ${ }^{40} \mathrm{~K}$ and ${ }^{14} \mathrm{C}$ ), and this contributes to our background radiation exposure. An X-ray beam is absorbed differently by different parts of the body, and these differences make shadows that are used to create an image or picture. A dense structure, such as bone, absorbs a high percentage of an X-ray beam (appears light grey on the image); whereas low-density structures, such as soft tissues (e.g. muscle and skin), absorb a small percentage (appears dark grey). Metal objects will usually show up as white and air will usually appear black. Man-made X-rays are electrically generated and are only present when the Xray machine is switched on. Once the $\mathrm{X}$-ray machine is switched off, there is no radiation coming from the X-ray machine. Computed tomography (CT) is a specialized X-ray 
examination using powerful computers to make the pictures. Having an X-ray or CT examination does not make a patient radioactive. The radiation exposure from having an X-ray, fluoroscopy, mammography or Computed Tomography (CT) examination only occurs while the machine is on and activated by the operator. Any operator of X-ray machines has to be trained in the use of these machines and holds a government license. Magnetic resonance imaging (MRI) uses strong magnetic fields and radio waves to make images. It does not use ionizing radiation. Ultrasound uses high frequency sound waves that the human ear cannot detect to make images. It does not use ionizing radiation. Although ultrasound imaging is generally considered safe when used prudently by appropriately trained health care providers, ultrasound energy has the potential to produce biological effects on the body. Ultrasound waves can heat the tissues slightly. In some cases, it can also produce small pockets of gas in body fluids or tissues (cavitation).

There are some technical research findings related to radiation exposure and it can be summarized as: (1) If the Radiation is greater than $5000 \mathrm{mSv}$ the patient will die between 2 hours (2) If the Radiation is (3000-4000) mSv the patient will die between 3 hours (3) If the Radiation is (5002000) $\mathrm{mSv}$ the patient will die between 1-3 days (4) It increases the cancer probability (5) It causes skin diseases, skin cancer and eye problem (6) It also creates the genetic problem, blood cancer and also the abortion in pregnant women (7) Cosmic ray which is primary, natural radiation (Uranium), Internal radiation source, Somatic effect (High range alpha particle) (8) Ionization particle cause cancer, lungs cancer, eye problem (9) A few parts of the world have background radiation 10 or more times greater than that generally found in Australia, but there are no studies that have shown an increased risk of cancer in populations living in areas with a higher background radiation level. (Hossian, 2009)

\subsection{Radiation Protection}

For occupational worker, the following precautions should be taken: (1) Minimum possible time of exposure and maximum possible distance should be maintained. (2) Only patient should be exposed to the primary beam. (3) Occupational workers should stay in the control panel which should be behind the X-ray tube and should have a protective wall or screen at least 7 feet high and provided with lead glass. (4) The exposure due to leakage radiation should not exceed $100 \mathrm{mr} / \mathrm{hr}$ at 1 meter from the focus. (5) Primary beam size should be small. (6) Reporting room should be away and behind the x-ray room. (7) Proper shielding of walls, floors and ceiling should be maintained. (8) Person should wear gloves with shisha (9) Suitable voltage limitation. (Hossian, 2009)

For patient, the following precautions should be taken: (1) Patient waiting for examination must be seated outside and away from the room. (2) The machine should be set up in proper side of the room. (3) Less time to get the picture. (4) Avoid unnecessary reexamination exposed patient. (5) X-ray of pregnant woman should be avoided as possible, especially during the time of organogenesis. (Hossian, 2009)

It has been found that most of the patients are not aware of the radiation and its effects and also how to take precautions. In addition, in some of the places, the operators were not also taking necessary precautions for taking data.

\section{CONCLUSION}

In this research, work was carried out to find the recent medical imaging condition of Bangladesh. Some data of imaging instruments were taken in Dhaka. It was found that some new techniques and instruments are not yet available in Bangladesh. Also, some hospitals and clinics have good number of new equipments available while some do not have adequate ones. The costs of some of the well-used equipments were taken. In future, we plan to take this data of all over the Bangladesh. There is another part of medical imaging like therapy. This work can be also extended to see the overview of medical therapeutic equipment conditions in Dhaka city as well as the other parts of Bangladesh. This is the overall view of biomedical imaging on the prospect of Bangladesh.

\section{REFERENCES}

[1] (Scatliff, 2014) J.H. Scatliff; P.J. Morris, 2014. From Röntgen to Magnetic Resonance Imaging: The History of Medical Imaging. NCMJ 75 (2), 111-113

[2] (Hodder, 2012) A. Hodder, 2012: Introduction to medical imaging. Imaging for StudentsDavid A Lisle. pp. 1-21.

[3] (Kunio, 2006) D.R. Kunio, 2006:Diagnostic imaging over the last 50 years: research and development in medical imaging science and technology. Phys. Med. Biol. 51, R5-R27

[4] (Smith-Bindman, 2008) R. Smith-Bindman; D.L. Miglioretti; E.B. Larson, 2008: Rising Use Of Diagnostic Medical Imaging In A Large Integrated Health System. Health Affairs, 27 (6), 1491-1502

[5] (Mueller, 1979) R.K. Mueller; M. Kaveh; G. Wade, 1979:Reconstructive tomography and applications to ultrasonics. Proc. IEEE, 67, 567-587

[6] (Duchene, 1985) B. Duchene; W. Tabbara, 1985:Tomographie ultrasonore par diffraction. Rev. Phys. Appl., 6, 299-304

[7] (Barber, 1983) D.C. Barber; B.H. Brown; I. L. Freeston, 1983: Imaging spatial distributions of resistivity using applied potential tomography.Electron. Left., 19 (22), 933-934

[8] (Bolomey, 1982) J.C. Bolomey; L. Jofre; Ch. Pichot;G. Peronnet,1982: Microwave diffraction tomography for biomedical applications. IEEE Trans. Microwave Theory Tech., MTT-30, 1988-2000.

[9] (Ermert, 1986) S.H. Ermert; M. Dohlus, 1986: Microwave-diffraction-tomography of cylindrical objects using 3-dimensional wave fields. ntzhchiv, Ed.Bd.8 H.5, 111-117

[10] (Nakata, 2014) S. Nakata, 2014: Universities in Bangladesh Making Strides Towards Home-Grown 
Innovations. MON, 04/14/2014 World Bank Group. http://blogs.worldbank.org/endpovertyinsouthasia/univ ersities-bangladesh-making-strides-towards-homegrown-innovations (accessed on 8.06.2016)

[11] (Hossian, 2009) M.F. Hossain, 2009: Radiological Physics, 106-107, Nisho's Publisher, $2^{\text {nd }}$ Edition, July 2009, Dhaka.

[12] (BD, 2016) Bangladesh Population. Website: http://www.worldometers.info/worldpopulation/bangladesh-population/ (accessed on 12-72016)

[13] (Dhaka, 2016) Dhaka City- Area Population. Media Bangladesh.

Website: http://www.mediabangladesh.net/dhaka-city-capital-ofbangladesh-area-population/ (accessed on 12-7-2016) 